\title{
Toward a New Geometric Distance to the Active Galaxy NGC 4258. II. Centripetal Accelerations and Investigation of Spiral Structure
}

\section{Citation}

Humphreys, E. M. L., Mark Jonathan Reid, Lincoln J. Greenhill, James M. Moran, and Alice L. Argon. 2008. "Toward a New Geometric Distance to the Active Galaxy NGC 4258. II. Centripetal Accelerations and Investigation of Spiral Structure." Astrophysical Journal 672 (2) (January 10): 800-816. doi:10.1086/523637.

\section{Published Version}

doi:10.1086/523637

\section{Permanent link}

http://nrs.harvard.edu/urn-3:HUL.InstRepos:11596973

\section{Terms of Use}

This article was downloaded from Harvard University's DASH repository, and is made available under the terms and conditions applicable to Other Posted Material, as set forth at http:// nrs.harvard.edu/urn-3:HUL.InstRepos:dash.current.terms-of-use\#LAA

\section{Share Your Story}

The Harvard community has made this article openly available.

Please share how this access benefits you. Submit a story.

Accessibility 
The Astrophysical Journal, 672:800-816, 2008 January 10

(C) 2008. The American Astronomical Society. All rights reserved. Printed in U.S.A.

\title{
TOWARD A NEW GEOMETRIC DISTANCE TO THE ACTIVE GALAXY NGC 4258. II. CENTRIPETAL ACCELERATIONS AND INVESTIGATION OF SPIRAL STRUCTURE
}

\author{
E. M. L. Humphreys, M. J. Reid, L. J. Greenhill, J. M. Moran, and A. L. Argon \\ Harvard-Smithsonian Center for Astrophysics, 60 Garden Street, Cambridge, MA 02138 \\ Received 2007 July 2; accepted 2007 September 6
}

\begin{abstract}
We report measurements of centripetal accelerations of maser spectral components of NGC 4258 for 51 epochs spanning 1994 to 2004. This is the second paper of a series, in which the goal is the determination of a new geometric maser distance to NGC 4258, accurate to possibly $\sim 3 \%$. We measure accelerations using a formal analysis method that involves simultaneous decomposition of maser spectra for all epochs into multiple, Gaussian components. Components are coupled between epochs by linear drifts (accelerations) from their centroid velocities at a reference epoch. For high-velocity emission, accelerations lie in the range -0.7 to $+0.7 \mathrm{~km} \mathrm{~s}^{-1} \mathrm{yr}^{-1}$, indicating an origin within $13^{\circ}$ of the disk midline (the perpendicular to the line of sight [LOS] to the black hole). Comparison of the projected positions of high-velocity emission in VLBI images with those derived from acceleration data provides evidence that masers trace real gas dynamics. High-velocity emission accelerations do not support a model of trailing shocks associated with spiral arms in the disk. However, we find strengthened evidence for spatial periodicity in highvelocity emission, of wavelength 0.75 mas. This supports suggestions of spiral structure due to density waves in the nuclear accretion disk of an active galaxy. Accelerations of low-velocity (systemic) emission lie in the range 7.7 to $8.9 \mathrm{~km} \mathrm{~s}^{-1} \mathrm{yr}^{-1}$, consistent with emission originating from a concavity where the thin, warped disk is tangent to the LOS. A trend in accelerations of low-velocity emission, as a function of Doppler velocity, may be associated with disk geometry and orientation or with the presence of spiral structure.
\end{abstract}

Subject headings: accretion, accretion disks — distance scale — galaxies: active — galaxies: nuclei — instabilities - masers

\section{INTRODUCTION}

The Hubble constant $\left(H_{0}\right)$ is a cornerstone of the extragalactic distance scale (EDS) and is a fundamental parameter of any cosmology. Measured period-luminosity (P-L) relations for Cepheid variable stars have been used to determine the EDS, based on the estimation of the distances to galaxies within $\sim 30 \mathrm{Mpc}$ and the calibration of distance indicators that are also found well into the Hubble flow (e.g., the Tully-Fisher relation and Type 1a supernovae). The best present estimate of $H_{0}$ (Freedman et al. 2001) is accurate to $10 \%, 72 \pm 3$ (random) \pm 7 (systematic) $\mathrm{km} \mathrm{s}^{-1} \mathrm{Mpc}^{-1}$. Other estimates of $H_{0}$ are generally consistent, although sometimes model dependent (e.g., microwave background fluctuations; Spergel et al. 2007) or somewhat outside the $\pm 10 \%$ uncertainties (e.g., $H_{0}=62 \mathrm{~km} \mathrm{~s}^{-1} \mathrm{Mpc}^{-1}$; Sandage et al. 2006 and references therein).

Several sources of systematic error affect the accuracy of Cepheid luminosity calibrations. These include uncertainty in the distance to the Large Magellanic Cloud (LMC), which determines the zero point of the P-L relation and for which recent estimates differ by up to $\pm 0.25 \mathrm{mag}$, or $\pm 12 \%$ (Benedict et al. 2002), and uncertainty in the impact of metallicity on the P-L relation (e.g., Udalski et al. 2001; Caputo et al. 2002; Jensen et al. 2003). The latter is particularly important because the LMC is metal-poor with respect to other galaxies in the Freedman et al. study. For a description of controversy concerning the estimation of $H_{0}$ see Macri et al. (2006) and Argon et al. (2007, hereafter Paper I).

For the nearby active galaxy NGC 4258, independent distances may be obtained from analysis of Cepheid brightness and water maser positions, velocities, and accelerations. Comparison of the distances would enable refinement of Cepheid calibrations and the supplementation or replacement of the LMC as an anchor, thus reducing uncertainty in $H_{0}$ and the EDS. Implications may include a better constraint of cosmological parameters, such as the flatness of the universe and the equation of state for dark energy (e.g., Hu 2005; Spergel et al. 2007), which would discriminate among different origins, e.g., the cosmological constant and quintessence.

Compact maser emission in NGC 4258, from the $616-5_{23}$ transition of water $(22,235.080 \mathrm{MHz})$ was first detected near the systemic velocity $\left(v_{\text {sys }}\right)$ by Claussen et al. (1984), where $v_{\text {sys }}=$ $472 \pm 4 \mathrm{~km} \mathrm{~s}^{-1}$ (referenced to the local standard of rest [LSR] and using the radio definition of Doppler shift; Cecil et al. 1992). The discovery of high-velocity emission at $\sim v_{\text {sys }} \pm 1000 \mathrm{~km} \mathrm{~s}^{-1}$ provided critical evidence that maser emission probably arises from material in orbit about a massive central black hole (Nakai et al. 1993). Early very long baseline interferometry (VLBI) observations showed that masers delineated an almost edge-on, subparsec-scale rotating disk (Greenhill et al. 1995a). Studies of maser centripetal accelerations, inferred from secular velocity drifts in the peaks of spectral components, produced independent evidence of a disk geometry (Haschick et al. 1994; Greenhill et al. 1995b; Nakai et al. 1995; Watson \& Wallin 1994). Low-velocity masers (near to $v_{\text {sys }}$ ) displayed positive drifts of $\sim 9 \mathrm{~km} \mathrm{~s}^{-1} \mathrm{yr}^{-1}$, which located them on the front side of the disk, whereas highvelocity masers drifted by $< \pm 1 \mathrm{~km} \mathrm{~s}^{-1} \mathrm{yr}^{-1}$, confining emission to lie close to the disk midline (the diameter perpendicular to the line of sight [LOS]). Very Long Baseline Array (VLBA) ${ }^{1}$ studies provided conclusive evidence supporting the disk model, established a Keplerian rotation curve to better than $1 \%$ accuracy for maser emission, and traced a warp in the disk structure (Miyoshi et al. 1995; Herrnstein et al. 1996, 2005; and early reviews of Moran et al. 1995, 1999).

\footnotetext{
1 The VLBA is operated by the National Radio Astronomy Observatory (NRAO), a facility of the National Science Foundation (NSF) operated under cooperative agreement by Associated Universities, Inc.
} 
A geometric distance can be obtained by modeling the threedimensional (3D) geometry and dynamics traced by the maser LOS velocities, positions, and LOS accelerations or proper motions. Assuming circular orbits, Herrnstein et al. (1999) obtained the most accurate distance to NGC 4258 thus far, $7.2 \pm$ 0.2 (random) \pm 0.5 (systematic) Mpc. The total error is $7 \%$, and the systematic component largely reflects an upper limit on the eccentricity of 0.1 for confocal particle orbits. This contributes $0.4 \mathrm{Mpc}$ of the systematic uncertainty budget.

This paper is the second of a series in which we revisit the task of estimating the maser distance to NGC 4258, using an expanded data set and more detailed analyses. Our goal is to reduce the uncertainty by a factor of $2-3$, through a reduction in both random and systematic errors. Herrnstein et al. (1999) used four epochs of VLBA data spanning $3 \mathrm{yr}$ to estimate distance. To this we have added 18 new VLBI epochs over 3.4 yr (Paper I). These combined data will facilitate more thorough disk modeling, including the incorporation of orbital eccentricity and periapsis angle as parameters. Here we address the measurement of radial accelerations for maser emission from a time-series of spectroscopic measurements, combining VLBI, Very Large Array (VLA), ${ }^{2}$ and single-dish spectra. Future work will present the model of the cumulative data (position, velocity, and acceleration) to obtain a best-fit distance.

In a parallel series of papers starting with Macri et al. (2006), analysis of recent three-color, wide-field Hubble Space Telescope (HST ) Cepheid photometry is undertaken to estimate an improved standard-candle distance to NGC 4258 (see Newman et al. 2001), with supplementary information from near-infrared and high angular resolution data that place tighter constraints on systematics (crowding and extinction).

Our approach to the measurement of accelerations is very different from that of previous work, which identified individual maser spectral Doppler components by local maxima in blended line profiles at each epoch. Components were matched up among different epochs on a maximum likelihood basis or in "by-eye" analyses, depending on the study, in order to determine drifts in component peak or fitted centroid velocities (Haschick et al. 1994; Greenhill et al. 1995b; Nakai et al. 1995; Herrnstein et al. 1999; Bragg et al. 2000). For low-velocity emission in particular, this method is subject to ambiguity in component identification unless the spacing between epochs is short ( $<3-4$ months), because of intensity fluctuations among blended, drifting components. This informal fitting technique is also susceptible to biases in the velocities of local maxima due to blending. Here we decompose the spectra into individual Gaussian components for many epochs simultaneously, constrained by a separate linear velocity drift in time for each feature. The constraint greatly increases the robustness of the decompositions, is less subjective than by-eye techniques, and enables the discernment of weak spectral features with greater confidence. Including the VLBI monitor data reported in Paper I, we applied this fitting to 36 spectra of low-velocity emission spanning $\approx 6 \mathrm{yr}$ and 40 spectra of high-velocity emission spanning $\approx 10 \mathrm{yr}$.

Implicit in the estimation of distance is the construction of a disk dynamical model from acceleration, position, and velocity measurements. These may also be used to detect disk substructure. Analysis of structure on the smallest scales enables identification of maser spots as representative of physical clumps. Clustering of maser spots enables tests of predicted spiral structure induced by gravitational instabilities. Maoz (1995) proposed that spiral density waves form in the NGC 4258 disk, and the

\footnotetext{
2 The VLA is operated by the NRAO, which is a facility of the NSF, operated under cooperative agreement by Associated Universities, Inc.
}

high-velocity masers mark the density maxima along the disk midline, which is where gain paths are greatest. Maoz \& McKee (1998) proposed that the spiral pattern is traced by shocks, with maser emission arising in relatively narrow postshock regions and visible only when the spiral arms are tangent to the LOS. Due to the pitch angle of the spiral pattern, the masers would arise systematically offset from the midline, and the acceleration signatures predicted by the two models enable discrimination (Bragg et al. 2000).

We describe the spectroscopic observations with which accelerations are estimated in $\S 2$. Decomposition of spectra into Gaussian components whose velocities drift in time and the inferred accelerations are described in $\S \S 3$ and 4. Comparisons with previous work are in $\S 5$. Implications of the new analyses for disk structure follow, with focus on the physical identification of maser clumps, signatures of spiral structure, and strong constraints on orbital eccentricity. The next paper in this series will give the improved distance to NGC 4258 estimated from analyses of maser data presented in Paper I and this paper.

\section{THE DATA SET}

The primary purpose of this paper is to report the measurements of maser component accelerations with high accuracy to be used in determination of a new geometric distance to NGC 4258. We attempted to reduce statistical uncertainties in the accelerations by including a larger number of epochs over a longer time baseline than was done by Herrnstein et al. (1999). In this study we analyzed spectra of water maser emission at $22.235 \mathrm{GHz}$ from NGC 4258 at 51 epochs, between 1994 April 19 and 2004 May 21. These data were presented in detail in Paper I. We summarize the observations in Table 1 and display subsets of the data in Figures 1 and 2 .

A compilation of the spectra measured from 1994 April 19 to 1997 February 10 was presented by Bragg et al. (2000). Observations were made approximately 1 to 2 months using the VLBA (5 epochs), the VLA (17 epochs), and the Effelsberg $100 \mathrm{~m}$ telescope of the Max-Planck-Institut für Radioastronomie (5 epochs). The $1 \sigma$ noise in the spectra range from 15 to $110 \mathrm{mJy}$ for channel spacings of $<0.35 \mathrm{~km} \mathrm{~s}^{-1}$ (Table 1). At the majority of epochs using the VLA and VLBA, both low- and high-velocity emission were measured, although some exceptions are noted in Table 1. Only the redshifted high-velocity spectrum was observed at Effelsberg.

The spectra from 18 VLBI epochs, measured between 1997 March 6 to 2000 August 12, are displayed in Paper I. Twelve of the epochs used the VLBA only (the "medium-sensitivity" epochs) and had $1 \sigma$ noise in the range 3.6 to $5.8 \mathrm{mJy}$ for $1.3 \mathrm{~km} \mathrm{~s}^{-1}$ wide channels. At medium-sensitivity epochs, low-velocity emission plus either redshifted or blueshifted high-velocity emission were observed. The observing setup was designed to enable exploration of a continuous range of velocities between known spectralline complexes and a limited range to higher velocities. The remaining six epochs (the "high-sensitivity" epochs) involved the VLBA, the phased VLA, and Effelsberg. The instantaneous bandwidth of the high-sensitivity observations was sufficient to perform simultaneous imaging of known low- and high-velocity emission, and resulted in $1 \sigma$ noise of 2.3-4.7 mJy per $\mathrm{km} \mathrm{s}^{-1}$ The average time between the observations was $\sim 2.5$ months. We extracted spectra from VLBI images by fitting two-dimensional Gaussian brightness distribution functions to all peaks in velocity channel maps. For a detailed description of the data analysis method, see Paper I. Of the remaining epochs, three were obtained using the Green Bank Telescope (GBT) between 2003 April 10 and December 8, with $1 \sigma$ noise levels of $\sim 3$ mJy per $0.16 \mathrm{~km} \mathrm{~s}^{-1}$ channel (Modjaz et al. 2005; M. Modjaz 2005, private communication; P. Kondratko 2006, private communication). 
TABLE 1

The Data Set

\begin{tabular}{|c|c|c|c|c|c|c|c|}
\hline & & & & & OBSERV & Details & \\
\hline & $\mathrm{OCH}$ & & OBSERVA & ONS & & & \\
\hline No. & Date & Day No. & Program Code & Telescope & $\left(\mathrm{km} \mathrm{s}^{-1}\right)$ & $(\mathrm{mJy})$ & Comments \\
\hline 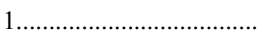 & 1994 Apr 19 & 0 & BM19 & VLBA & 0.21 & 43 & Systemic, red- and blueshifted emission \\
\hline 2 & 1995 Jan 7 & 263 & AG448 & VLA-CD & 0.33 & 25 & Systemic, red- and blueshifted emission \\
\hline 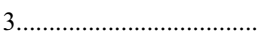 & 1995 Jan 8 & 264 & BM36а & VLBA & 0.21 & 80 & Systemic, red- and blueshifted emission \\
\hline 4 & 1995 Feb 23 & 310 & AG448 & VLA-D & 0.33 & 25 & Systemic, red- and blueshifted emission \\
\hline 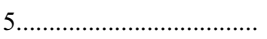 & 1995 Mar 16 & 331 & $\ldots$ & EFLS & 0.33 & 85 & Redshifted emission only \\
\hline 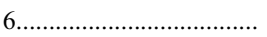 & 1995 Mar 24 & 339 & $\ldots$ & EFLS & 0.33 & 90 & Redshifted emission only \\
\hline ...................... & 1995 Mar 25 & 340 & $\ldots$ & EFLS & 0.33 & 95 & Redshifted emission only \\
\hline 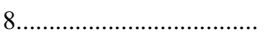 & 1995 Apr 4 & 350 & $\ldots$ & EFLS & 0.33 & 85 & Redshifted emission only \\
\hline 9 & 1995 Apr 20 & 366 & AG448 & VLA-D & 0.33 & 20 & Systemic, red- and blueshifted emission \\
\hline 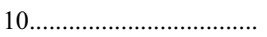 & 1995 May 29 & 405 & BM36b & VLBA & 0.21 & 30 & Systemic, red- and blueshifted emission \\
\hline ............. & 1995 Jun 8 & 415 & AG448 & VLA-AD & 0.33 & 20 & Systemic, red- and blueshifted emission \\
\hline $12 \ldots \ldots$ & 1995 Jun 25 & 432 & $\ldots$ & EFLS & 0.33 & 110 & Redshifted emission only \\
\hline 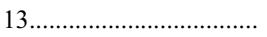 & 1995 Jul 29 & 466 & AG448 & VLA-A & 0.33 & $\ldots$ & No data \\
\hline 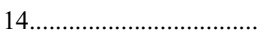 & 1995 Sep 9 & 508 & AG448 & VLA-AB & 0.33 & $\ldots$ & No data \\
\hline 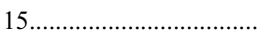 & 1995 Nov 9 & 569 & AG448 & VLA-B & 0.33 & $37-60$ & Systemic, red- and blueshifted emission \\
\hline 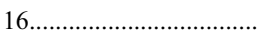 & 1996 Jan 11 & 632 & AG448 & VLA-BC & 0.33 & 20 & Systemic, red- and blueshifted \\
\hline 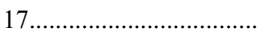 & 1996 Feb 22 & 674 & BM56a & VLBA & 0.21 & 25 & Systemic, red- and blueshifted emission \\
\hline 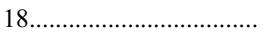 & 1996 Feb 26 & 678 & AG448 & VLA-C & 0.33 & $23-30$ & Systemic, red- and blueshifted emission \\
\hline 19 & 1996 Mar 29 & 710 & AG448 & VLA-C & 0.33 & 20 & Systemic, red- and blueshifted emission \\
\hline 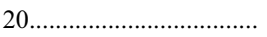 & 1996 May 10 & 752 & AG448 & VLA-CD & 0.33 & $15-24$ & Systemic, red- and blueshifted emission \\
\hline $21 \ldots \ldots \ldots \ldots$ & 1996 Jun 27 & 799 & AG448 & VLA-D & 0.33 & $\ldots$ & No data \\
\hline $22 \ldots \ldots \ldots \ldots \ldots$ & 1996 Aug 12 & 846 & AG448 & VLA-D & 0.33 & $23-36$ & Systemic, red- and blueshifted emission \\
\hline 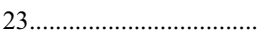 & 1996 Sep 21 & 886 & BM56b & VLBA & 0.21 & 20 & High-velocity emission only \\
\hline 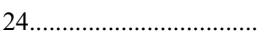 & 1996 Oct 3 & 898 & AG448 & VLA-AD & 0.33 & 50 & Systemic, red- and blueshifted emission \\
\hline $25 \ldots \ldots \ldots$ & 1996 Nov 21 & 947 & AG448 & VLA-A & 0.33 & $21-26$ & Systemic and redshifted emission \\
\hline 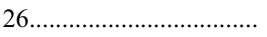 & 1997 Jan 20 & 1007 & AG448 & VLA-AB & 0.33 & $27-37$ & Systemic and redshifted emission \\
\hline 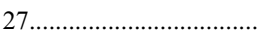 & 1997 Feb 10 & 1028 & AG448 & VLA-AB & 0.33 & $17-23$ & Systemic and redshifted emission \\
\hline 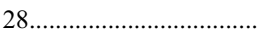 & 1997 Mar 6 & 1052 & BM56c & VLBA & 0.21 & 4.7 & Systemic, red- and blueshifted emission \\
\hline 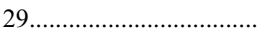 & 1997 Oct 1 & 1261 & BM81a & VLBA & 0.21 & 4.1 & Systemic, red- and blueshifted emission \\
\hline 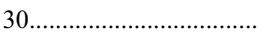 & 1998 Jan 27 & 1379 & BM81b & VLBA & 0.21 & 5.0 & Systemic, red- and blueshifted emission \\
\hline 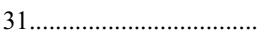 & 1998 Sep 5 & 1600 & BM112a & VLBA & 0.21 & 4.6 & Systemic, red- and blueshifted emission \\
\hline 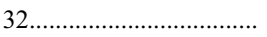 & 1998 Oct 18 & 1643 & BM112b & VLBA & 0.42 & 4.6 & Systemic and redshifted emission \\
\hline 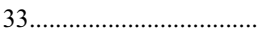 & 1998 Nov 16 & 1672 & BM112c & VLBA & 0.42 & 4.4 & Systemic and blueshifted emission \\
\hline 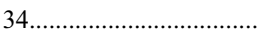 & 1998 Dec 24 & 1710 & BM112d & VLBA & 0.42 & $\ldots$ & No data \\
\hline 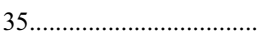 & 1999 Jan 28 & 1745 & BM112e & VLBA & 0.42 & 4.8 & Systemic and blueshifted emission \\
\hline 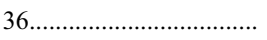 & 1999 Mar 19 & 1795 & BM112f & VLBA & 0.42 & 4.5 & Systemic and redshifted emission \\
\hline 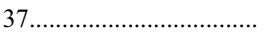 & 1999 May 18 & 1855 & BM112g & VLBA & 0.42 & 5.2 & Systemic and blueshifted emission \\
\hline 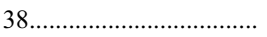 & 1999 May 26 & 1863 & BM112h & VLBA & 0.21 & 3.5 & Systemic, red- and blueshifted emission \\
\hline 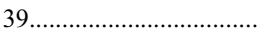 & 1999 Jul 15 & 1913 & BM112i & VLBA & 0.42 & $\ldots$ & No data \\
\hline 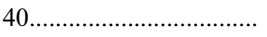 & 1999 Sep 15 & 1975 & BM112j & VLBA & 0.42 & 6.3 & Systemic and blueshifted emission \\
\hline 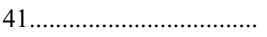 & 1999 Oct 29 & 2019 & BM112k & VLBA & 0.42 & 4.7 & Systemic and blueshifted emission \\
\hline 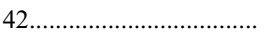 & 2000 Jan 7 & 2089 & BM1121 & VLBA & 0.42 & 3.9 & Systemic and blueshifted emission \\
\hline 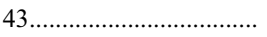 & 2000 Jan 30 & 2112 & $\mathrm{BM} 112 \mathrm{~m}$ & VLBA & 0.42 & 4.7 & Systemic and redshifted emission \\
\hline 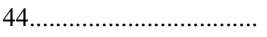 & 2000 Mar 04 & 2146 & BM112n & VLBA & 0.42 & 4.3 & Systemic and blueshifted emission \\
\hline 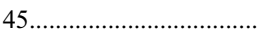 & 2000 Apr 12 & 2185 & BM112o & VLBA & 0.42 & 5.5 & Systemic and redshifted emission \\
\hline 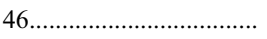 & 2000 May 04 & 2207 & BM112p & VLBA & 0.42 & 5.0 & Systemic and blueshifted emission \\
\hline 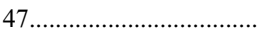 & 2000 Aug 12 & 2307 & BG107 & VLBA & 0.42 & 7.2 & Systemic, red- and blueshifted emission \\
\hline 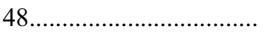 & 2003 Apr 10 & 3298 & $\ldots$ & GBT & 0.21 & 2.9 & Systemic, red- and blueshifted emission \\
\hline 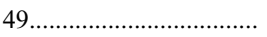 & 2003 Oct 23 & 3474 & $\ldots$ & GBT & 0.21 & 2.9 & Systemic, red- and blueshifted emission \\
\hline 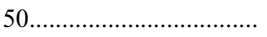 & 2003 Dec 8 & 3520 & $\ldots$ & GBT & 0.21 & 2.9 & Systemic, red- and blueshifted emission \\
\hline 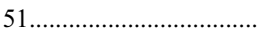 & 2004 May 21 & 3685 & AH847 & VLA & 0.21 & 20 & Portions of redshifted spectrum \\
\hline
\end{tabular}

${ }^{\text {a }}$ Channel spacing and sensitivities (rms) for epochs 1-27 are taken from Bragg et al. (2000), for 48-47 from Paper I, and for GBT data from M. Modjaz \& P. Kondratko (2007, private communications).

We obtained the final epoch of data on 2004 May 21 using the VLA, for limited portions of the redshifted high-velocity spectrum only and with an rms noise of $20 \mathrm{mJy}$ per $0.21 \mathrm{~km} \mathrm{~s}^{-1}$ channel.

\section{MULTIEPOCH SPECTRUM DECOMPOSITION}

To measure accelerations of maser components, as well as amplitude and line width variations during the monitoring, we decomposed portions of spectra from multiple epochs simultaneously into individual Gaussian line profiles. We identified a component at different epochs by solving for its constant drift from a centroid velocity at a reference time. We could therefore account for each Gaussian at every epoch, even if the amplitude had fallen below our detection threshold, which minimized potential for confusion among components. 

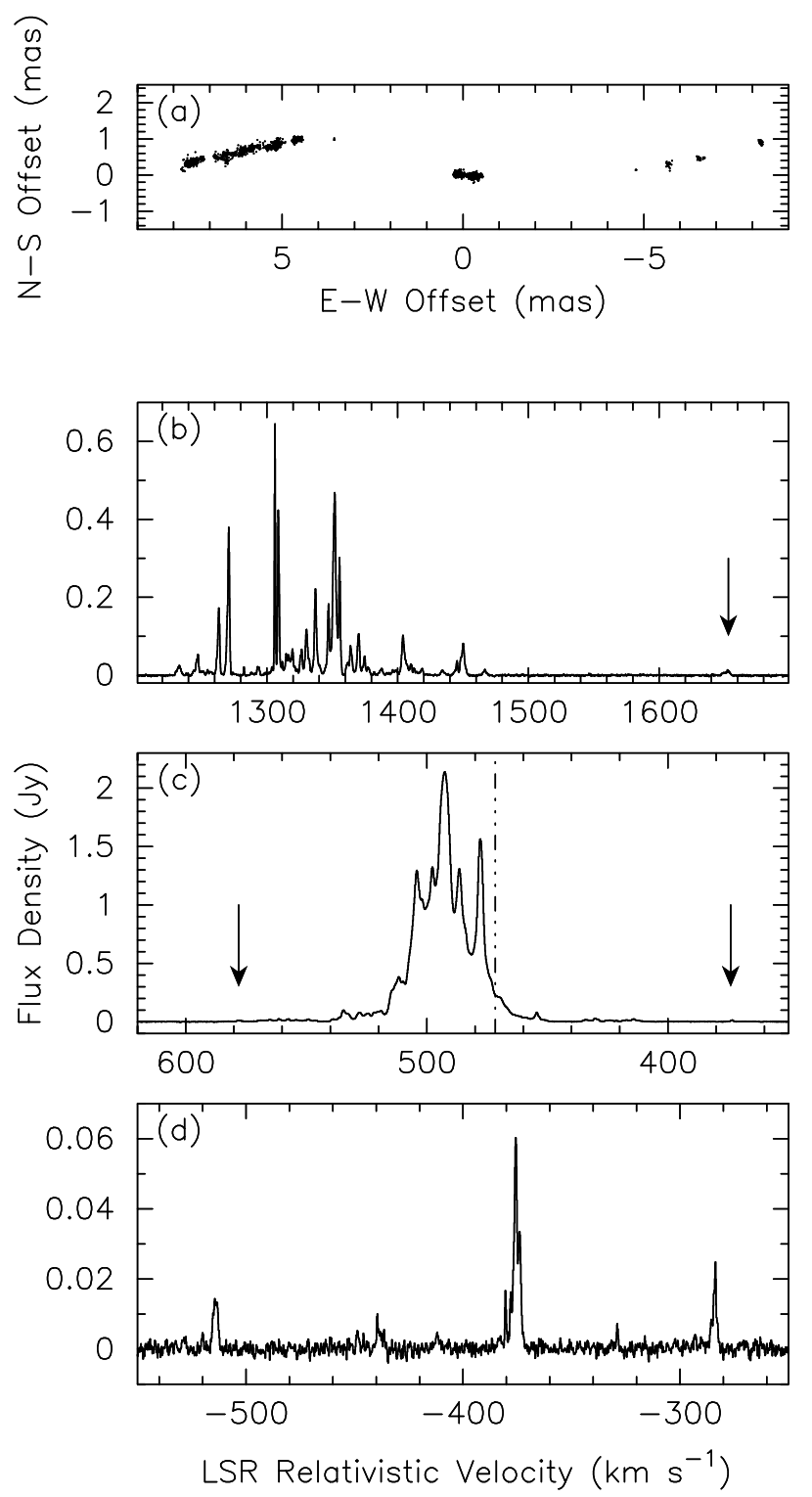

FIG. 1.- (a) Sky positions for $22 \mathrm{GHz}$ maser emission in NGC 4258 obtained using VLBI on 18 epochs from 1997 March 6 to 2000 August 12 described in Paper I. Redshifted high-velocity emission occurs from 3 to 8 mas in the disk; low-velocity emission occurs in the range -1 to 1 mas and blueshifted high-velocity emission in the range $5-9$ mas. Note that $(0,0)$ in the plot does not represent the disk dynamical center. (b) GBT spectrum of redshifted high-velocity emission from Modjaz et al. (2005) obtained on 2003 October 23. The $1 \sigma$ noise in the spectrum is $1.6 \mathrm{mJy}$. The arrow marks weak, newly detected emission at $1652 \mathrm{~km} \mathrm{~s}^{-1}$ (Paper I). (c) GBT spectrum of low-velocity maser emission. The arrows mark emission extrema. The dotted line denotes the galactic systemic velocity of $472 \mathrm{~km} \mathrm{~s}^{-1}$ (Cecil et al. 1992). (d) GBT spectrum of blueshifted maser emission.

We used a nonlinear, multiple Gaussian-component leastsquares $\chi^{2}$ minimization routine that we dimensioned for a maximum of 84 time-varying Gaussians and 40 epochs of data in any given fit, which was limited by computational factors. Each maser component was represented by a Doppler velocity $v_{\text {los }}\left(t_{0}\right)$ at a reference time $t_{0}$, a linear velocity drift $\dot{v}_{\text {los }}$, and a line width and amplitude at every epoch. We could choose to solve for either constant or time-varying component line widths. We set a priori line widths to prevent the large deviations from physical values expected for maser line widths at gas kinetic temperatures of 400 to $1000 \mathrm{~K}$. The a priori determinations were included as extra
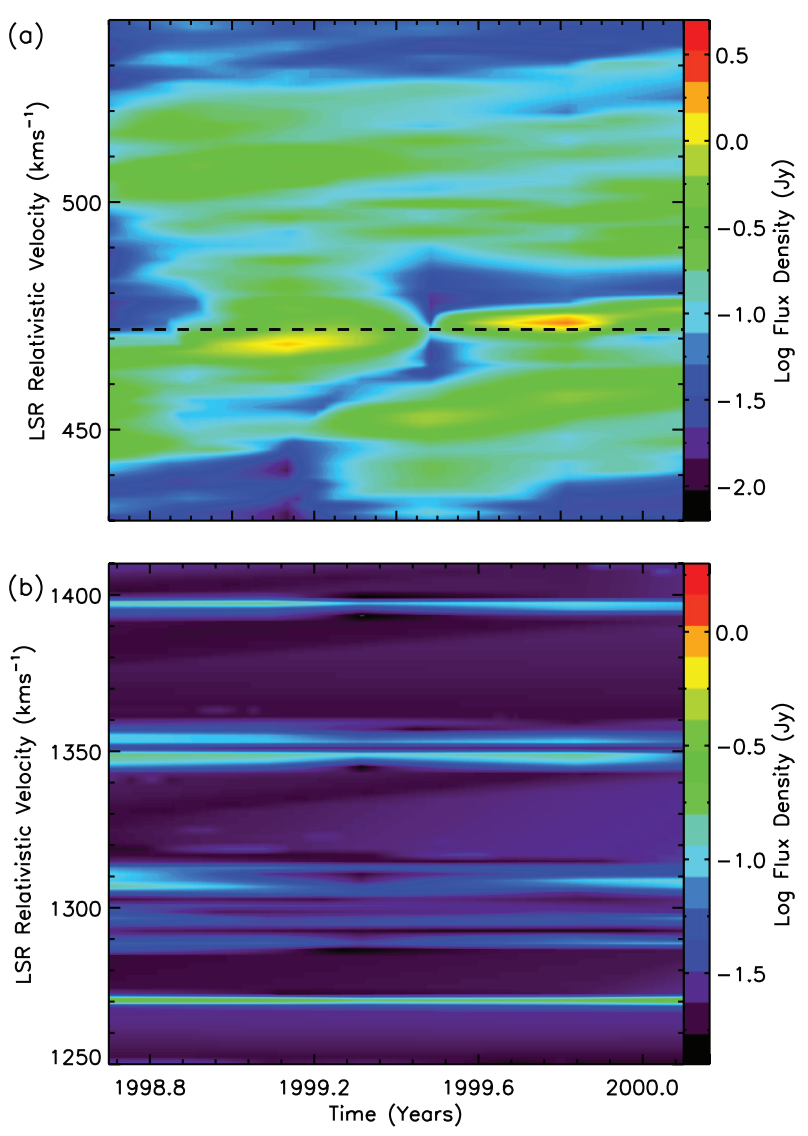

Fig. 2.- (a) Velocity vs. time plot for systemic components using data from 1998 January 27 to 2000 August 12, during which sampling was every $\sim 3$ months. Drifts in Doppler velocities of strong components are clearly evident and consistent with centripetal accelerations. The dashed line marks the galactic systemic velocity of $472 \mathrm{~km} \mathrm{~s}^{-1}$. (b) Corresponding plot for redshifted components. Small magnitudes of drifts in component Doppler velocities are consistent with near-zero observed accelerations for components near the disk midline.

data points in the fit, and typically prevented deviations of greater than 4 times the a priori uncertainties. The reference time was chosen to be near the middle of the monitoring period. All velocities in this paper are quoted for a $t_{0}$ of 1999 October 10 , or monitoring day 2000, unless stated otherwise.

We note that the fitting of linear velocity drifts is an approximation for particles on circular orbits in a disk in which $v_{\text {los }}=$ $v_{\text {rot }} \cos \left(\Omega \Delta t+\phi_{0}\right)+v_{\text {sys }}$, where $v_{\text {rot }}$ is the rotational velocity at any given $r, \Omega$ is the angular velocity, $\Delta t=t-t_{0}$, and $\phi_{0}$ is the angle from the midline at a reference time $t_{0}$. From Monte Carlo simulations, we estimate that the maximum error we introduce by using this approximation is $0.1 \mathrm{~km} \mathrm{~s}^{-1} \mathrm{yr}^{-1}$. We chose the linear approximation in order to determine velocity drifts without any model assumptions.

\subsection{High-Velocity Emission}

The high-velocity spectrum consists of isolated blends of small numbers of components with low drift rates (Figs. 1 and 2). To facilitate the decomposition, we divided the high-velocity spectra into individual blends, of typical velocity extent $10-20 \mathrm{~km} \mathrm{~s}^{-1}$. We performed the fitting of each blend iteratively. First, we identified the number of prominent peaks in the blend at a highsensitivity and high spectral resolution epoch (e.g., 1998 September 5). We fit this number of Gaussian functions to the data 
TABLE 2

Fitted Accelerations For Redshifted High-Velocity Doppler Components

\begin{tabular}{|c|c|c|c|c|c|c|}
\hline \multicolumn{3}{|c|}{ CoMPONENT } & \multicolumn{2}{|c|}{ Ероснs } & \multirow[b]{2}{*}{$\begin{array}{c}\text { Time-Averaged Azimuth Angle } \\
(\mathrm{deg})\end{array}$} & \multirow[b]{2}{*}{$\begin{array}{c}\text { COMPONENT RADIUS } \\
(\mathrm{pc})\end{array}$} \\
\hline No. & $\begin{array}{l}\text { Velocity } \\
\left(\mathrm{km} \mathrm{s}^{-1}\right)\end{array}$ & $\begin{array}{l}\text { Acceleration } \\
\left(\mathrm{km} \mathrm{s}^{-1} \mathrm{yr}^{-1}\right)\end{array}$ & No. in Fit & $\begin{array}{l}\text { Time Baseline } \\
(\mathrm{yr})\end{array}$ & & \\
\hline 1. & $1248.14 \pm 0.02$ & $-0.06 \pm 0.02$ & 24 & 6.0 & $-1.6 \pm 0.6$ & 0.28 \\
\hline 2 & $1250.80 \pm 0.03$ & $-0.35 \pm 0.03$ & 24 & 6.0 & $-8.9 \pm 0.8$ & 0.28 \\
\hline 3 & $1252.24 \pm 0.01$ & $0.17 \pm 0.01$ & 24 & 6.0 & $4.3 \pm 0.3$ & 0.28 \\
\hline 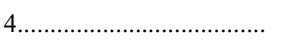 & $1254.34 \pm 0.02$ & $-0.01 \pm 0.03$ & 24 & 6.0 & $-0.3 \pm 0.7$ & 0.28 \\
\hline 5 & $1270.89 \pm 0.05$ & $0.11 \pm 0.04$ & 24 & 6.0 & $2.5 \pm 1.0$ & 0.27 \\
\hline 6 & $1282.21 \pm 0.06$ & $-0.15 \pm 0.08$ & 4 & 0.7 & $-3.1 \pm 1.8$ & 0.26 \\
\hline 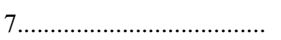 & $1283.87 \pm 0.08$ & $-0.05 \pm 0.14$ & 4 & 0.7 & $-1.1 \pm 3.0$ & 0.26 \\
\hline 8 & $1309.08 \pm 0.01$ & $-0.09 \pm 0.01$ & 30 & 9.0 & $-1.7 \pm 0.1$ & 0.24 \\
\hline 9 & $1328.64 \pm 0.04$ & $0.73 \pm 0.02$ & 29 & 5.3 & $12.6 \pm 0.6$ & 0.23 \\
\hline 10 & $1330.73 \pm 0.06$ & $0.73 \pm 0.03$ & 29 & 5.3 & $12.4 \pm 0.7$ & 0.23 \\
\hline $11 \ldots$ & $1337.36 \pm 0.04$ & $-0.16 \pm 0.03$ & 35 & 6.3 & $-2.7 \pm 0.5$ & 0.23 \\
\hline 12 & $1339.58 \pm 0.01$ & $-0.28 \pm 0.01$ & 35 & 6.3 & $-4.6 \pm 0.3$ & 0.22 \\
\hline $13 \ldots \ldots \ldots \ldots \ldots \ldots \ldots \ldots \ldots \ldots . .$. & $1351.00 \pm 0.03$ & $0.40 \pm 0.03$ & 34 & 6.0 & $6.1 \pm 0.5$ & 0.22 \\
\hline $14 \ldots \ldots \ldots \ldots \ldots \ldots \ldots \ldots$ & $1353.85 \pm 0.03$ & $0.11 \pm 0.03$ & 34 & 6.0 & $1.8 \pm 0.4$ & 0.22 \\
\hline $15 \ldots \ldots \ldots \ldots \ldots \ldots \ldots \ldots \ldots$ & $1355.56 \pm 0.02$ & $-0.10 \pm 0.02$ & 34 & 6.0 & $-1.5 \pm 0.3$ & 0.22 \\
\hline 16 & $1395.59 \pm 0.09$ & $0.24 \pm 0.05$ & 21 & 4.3 & $3.1 \pm 0.7$ & 0.20 \\
\hline 17 & $1398.09 \pm 0.05$ & $0.58 \pm 0.03$ & 21 & 4.3 & $7.3 \pm 0.4$ & 0.20 \\
\hline 18 & $1403.60 \pm 0.01$ & $-0.05 \pm 0.02$ & 34 & 6.0 & $-0.6 \pm 0.2$ & 0.19 \\
\hline 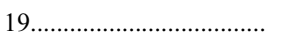 & $1406.74 \pm 0.05$ & $-0.06 \pm 0.04$ & 34 & 6.0 & $-0.8 \pm 0.5$ & 0.19 \\
\hline 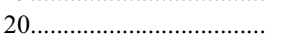 & $1449.43 \pm 0.04$ & $0.30 \pm 0.04$ & 25 & 2.9 & $3.0 \pm 0.5$ & 0.18 \\
\hline $21 \ldots$ & $1452.35 \pm 0.01$ & $-0.40 \pm 0.02$ & 25 & 2.9 & $-4.0 \pm 0.2$ & 0.18 \\
\hline 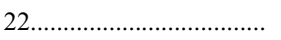 & $1452.80 \pm 0.14$ & $0.04 \pm 0.06$ & 25 & 2.9 & $0.4 \pm 0.6$ & 0.18 \\
\hline 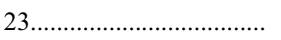 & $1455.26 \pm 0.19$ & $-0.33 \pm 0.09$ & 25 & 2.9 & $-3.2 \pm 0.9$ & 0.17 \\
\hline $24 \ldots$ & $1468.08 \pm 0.07$ & $0.34 \pm 0.08$ & 4 & 2.6 & $3.2 \pm 0.8$ & 0.17 \\
\hline
\end{tabular}

${ }^{\text {a }}$ Component velocities (relativistic definition) for fits that met requirements of $\S 3.1$ are quoted for 1999 October 10 , day 2000 of our monitoring campaign. Uncertainties are the $1 \sigma$ errors scaled by $\chi^{2}$ per degree of freedom.

${ }^{\mathrm{b}}$ Uncertainties are the $1 \sigma$ errors scaled by $\chi^{2}$ per degree of freedom.

${ }^{\mathrm{c}}$ Calculated using a black hole mass of $3.8 \times 10^{7} M_{\odot}$ (Herrnstein et al. 2005) for a flat disk.

${ }^{\mathrm{d}}$ Quantities are the weighted mean and the weighted deviation from the mean.

over all epochs and examined the residuals at each epoch. Where deviations in the residuals exceeded $5 \sigma$, we refit the segment using more Gaussian functions. We repeated this procedure until there was no systematic structure remaining in the residuals above the $5 \sigma$ level. We solved for constant line widths as a function of time for high-velocity emission, because the signal was typically $<1 \mathrm{Jy}$, and line widths at any given epoch were otherwise not well constrained. The fits for each high-velocity blend included between 4 and 40 epochs of data, covering time baselines of 0.5 to 9 yr (Tables 2 and 3) depending on component lifetime and time sampling. Example fits are shown in Figures 3 and 4 for red- and blueshifted emission, respectively.

\subsection{Low-Velocity Emission}

The low-velocity emission spectrum consists of strong (typically 2 to $10 \mathrm{Jy}$ ), highly blended emission for which previous work has shown that components drift at a mean rate of $\sim 9 \mathrm{~km} \mathrm{~s}^{-1} \mathrm{yr}^{-1}$ (Haschick et al. 1994; Greenhill et al. 1995b; Nakai et al. 1995; Herrnstein et al. 1999; Bragg et al. 2000). Some studies note a systematic trend in accelerations of low-velocity emission as a function of Doppler velocity, with accelerations of $8.6 \pm$ $0.5 \mathrm{~km} \mathrm{~s}^{-1} \mathrm{yr}^{-1}$ at velocities less than $470 \mathrm{~km} \mathrm{~s}^{-1}$ and of $10.3 \pm$ $0.6 \mathrm{~km} \mathrm{~s}^{-1} \mathrm{yr}^{-1}$ at velocities greater than $470 \mathrm{~km} \mathrm{~s}^{-1}$ (Haschick et al. 1994; Greenhill et al. 1995b). Others do not draw attention to such an effect (Nakai et al. 1995; Herrnstein et al. 1999; Bragg et al. 2000). We therefore approached decomposition of the lowvelocity spectra using two methods: (1) assuming that the linear velocity drift approximation holds over the entire 36 epoch monitoring and including all 36 epochs of data; and (2) assuming that there may be changes in accelerations during the monitoring period and dividing the data in four consecutive data sets of nine epochs for which fitting was performed independently. When weighting the data for fitting, we added $2 \%$ of the channel flux density in quadrature to the $1 \sigma$ noise to account for the dynamic range limitations of our observations (see Paper I).

To perform the 36-epoch fitting, we first decomposed the spectrum into a preliminary set of Gaussian components at one epoch only. We selected a high spectral resolution and high-sensitivity epoch for this purpose (1998 September 5). We performed a fit over a subset of six adjacent epochs for the low-velocity spectrum as a whole to obtain preliminary values of acceleration. We increased the number of Gaussian functions used in the fitting with each successive iteration, adding additional Gaussians to the fit, until no $5 \sigma$ systematic deviations remained (in the residuals). This analysis provided a set of "seed" parameters with which to perform a new decomposition over 36 epochs. For ease of computation, we divided the spectrum into segments of $\sim 12$ Gaussian components and performed the decomposition of each segment iteratively. We used a moving time window to select the relevant data range for segments at different epochs to track the $\sim 9 \mathrm{~km} \mathrm{~s}^{-1} \mathrm{yr}^{-1}$ accelerations. At segment edges, we overlapped fitted regions in each case by two to three components to ensure that fits were consistent across the low-velocity spectrum. Using this method, we obtained residual deviations of $<5 \sigma$ everywhere except at the 
TABLE 3

Fitted Accelerations For Blueshifted High-Velocity Doppler Components

\begin{tabular}{|c|c|c|c|c|c|c|}
\hline \multicolumn{3}{|c|}{ COMPONENT } & \multicolumn{2}{|c|}{ ЕроснS } & \multirow[b]{2}{*}{$\begin{array}{c}\text { Time-Averaged Azimuth Angle } \\
\text { (deg) }\end{array}$} & \multirow[b]{2}{*}{$\begin{array}{c}\text { Component Radius } \\
(\mathrm{pc})\end{array}$} \\
\hline No. & $\begin{array}{l}\text { Velocity }{ }^{\mathrm{a}, \mathrm{b}} \\
\left(\mathrm{km} \mathrm{s}^{-1}\right)\end{array}$ & $\begin{array}{l}\text { Acceleration }{ }^{\mathrm{b}} \\
\left(\mathrm{km} \mathrm{s}^{-1} \mathrm{yr}^{-1}\right)\end{array}$ & No. in Fit & $\begin{array}{l}\text { Time Baseline } \\
\text { (yr) }\end{array}$ & & \\
\hline 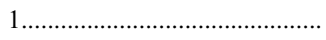 & $-282.21 \pm 0.07$ & $-0.47 \pm 0.10$ & 6 & 1.5 & $-13.2 \pm 2.9$ & 0.29 \\
\hline 2 & $-284.00 \pm 0.06$ & $-0.12 \pm 0.08$ & 6 & 1.5 & $-3.3 \pm 2.3$ & 0.29 \\
\hline 3.1-1 & $-286.05 \pm 0.13$ & $-0.38 \pm 0.13$ & 6 & 1.5 & $-10.3 \pm 3.5$ & 0.29 \\
\hline 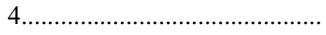 & $-374.20 \pm 0.05$ & $-0.32 \pm 0.26$ & 5 & 1.1 & $-5.6 \pm 4.7$ & 0.23 \\
\hline 5 & $-375.78 \pm 0.22$ & $-0.72 \pm 0.49$ & 5 & 1.1 & $-12.6 \pm 8.6$ & 0.23 \\
\hline 6 & $-435.00 \pm 0.03$ & $-0.29 \pm 0.05$ & 16 & 3.5 & $-3.9 \pm 0.7$ & 0.20 \\
\hline 7 & $-439.97 \pm 0.02$ & $+0.04 \pm 0.04$ & 15 & 2.9 & $0.5 \pm 0.5$ & 0.20 \\
\hline 8 & $-514.48 \pm 0.04$ & $-0.24 \pm 0.16$ & 4 & 0.5 & $-2.3 \pm 1.5$ & 0.17 \\
\hline Mean Acceleration: ${ }^{\mathrm{d}}$ & & $-0.21 \pm 0.08$ & Mean Azir & h Angle: ${ }^{d}$ & $-3.3 \pm 6.9$ & \\
\hline
\end{tabular}

${ }^{a}$ Component velocities (relativistic definition) are quoted for 1999 October 10, day 2000 of our monitoring campaign. Uncertainties are the $1 \sigma$ errors scaled by $\chi^{2}$ per degree of freedom.

${ }^{b}$ Uncertainties are the $1 \sigma$ errors scaled by $\chi^{2}$ per degree of freedom.

${ }^{c}$ Calculated using a black hole mass of $3.8 \times 10^{7} M_{\odot}$ (Herrnstein et al. 2005) for a flat disk.

${ }^{d}$ Quantities are the weighted mean and the weighted deviation from the mean.

time extrema of our data set, where we obtained significantly larger deviations in residuals.

For the 9-epoch fits, we also decomposed the spectrum into a preliminary set of Gaussian components at one epoch only (chosen to be near the middle of each data set). We then performed an initial fit over the entire nine epochs to obtain component accelerations. Unlike the 36-epoch fitting, it was not necessary to subdivide the spectrum and use a moving time window, and we performed the decomposition across the low-velocity spectrum as a whole in each case. We increased the number of Gaussians in

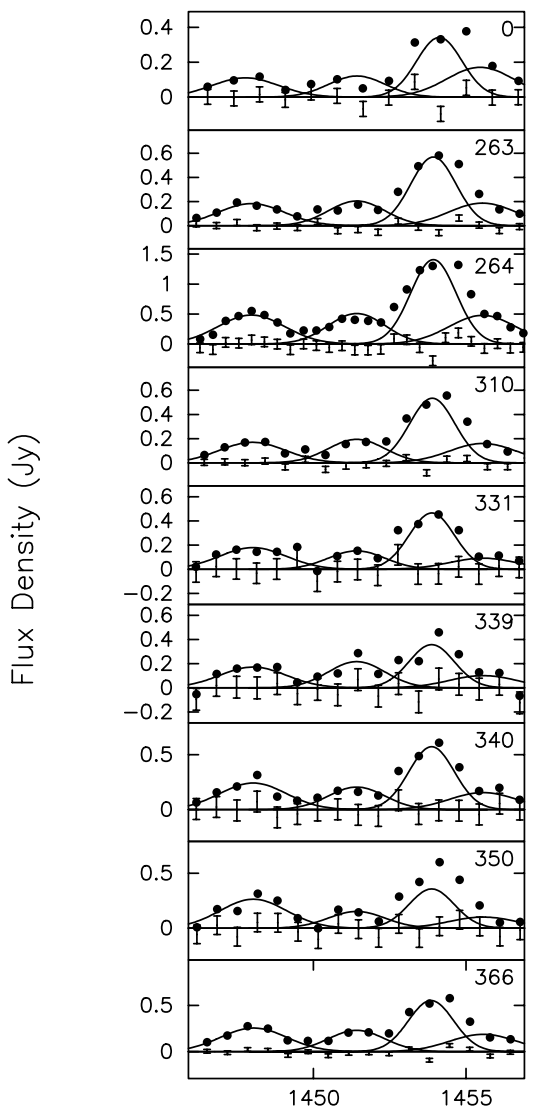

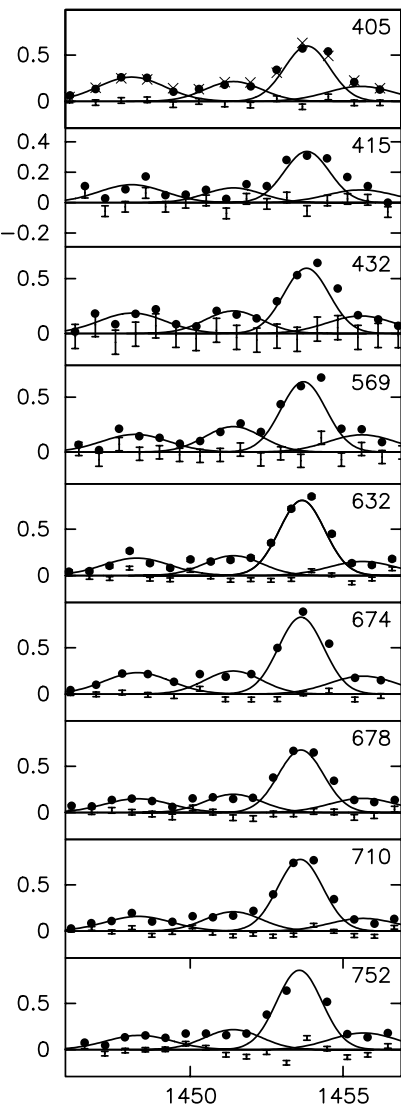

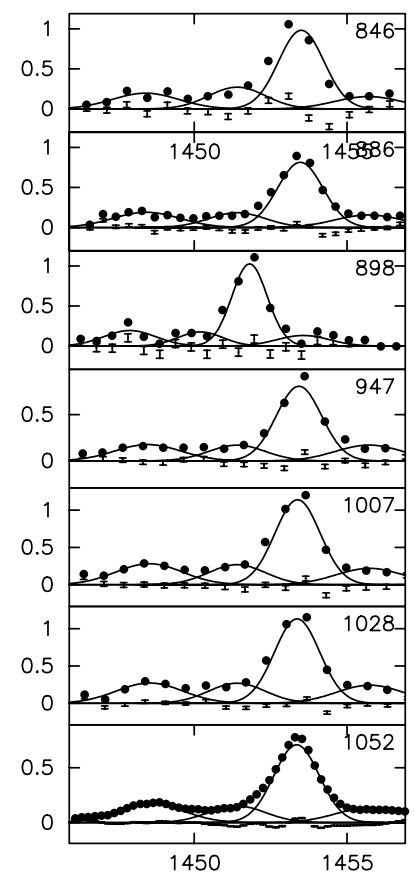

LSR Relativistic Velocity $\left(\mathrm{km} \mathrm{s}^{-1}\right)$

FIG. 3.-Example of a simultaneous four-component Gaussian function fit to a blend of redshifted components over 25 epochs (2.9 yr). Filled circles mark the data points. Residuals are plotted with $1 \sigma$ error bars. Model Gaussian functions are shown as solid black lines. The epoch day number is marked in the top right of each panel. Table 1 lists the corresponding dates. 


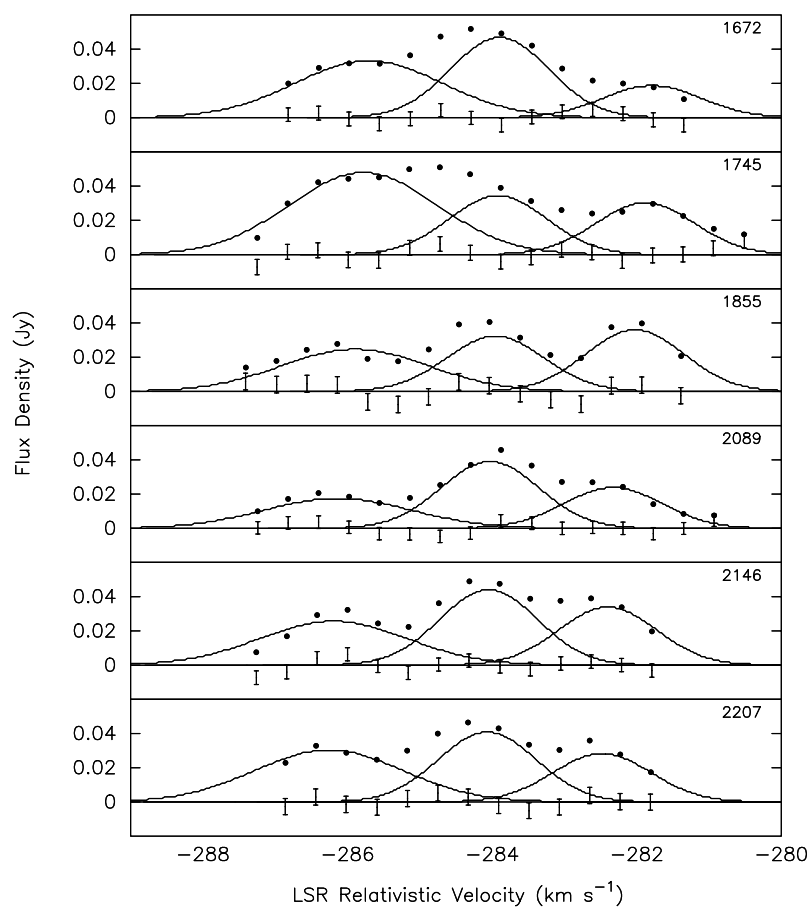

FIG. 4.- Same as Fig. 3, but for a blueshifted high-velocity emission blend.

each of the fits iteratively, examining the residuals after each fit and adding components where large deviations occurred. We repeated this process until no $>5 \sigma$ systematic deviations remained in the residuals. In each of the 9-epoch fits, this required $\sim 55$ Gaussians of line width $1-4 \mathrm{~km} \mathrm{~s}^{-1}$. The four independent fits to the data indicated that there is a persistent trend of component accelerations as a function of Doppler velocity (or equivalently time), explaining why a good fit was not obtained in the longer time baseline, 36-epoch method. We adopt results from the 9-epoch fits (e.g., Fig. 5) in the sections that follow.

\section{RESULTS}

\subsection{High-Velocity Emission}

We measured accelerations for 24 red- and 8 blueshifted highvelocity components and found them to range between -0.40 and $+0.73 \mathrm{~km} \mathrm{~s}^{-1} \mathrm{yr}^{-1}$ and between -0.72 and $+0.04 \mathrm{~km} \mathrm{~s}^{-1} \mathrm{yr}^{-1}$, respectively (Tables 2 and 3). We found a weighted average of redshifted high-velocity accelerations of $0.02 \pm 0.06 \mathrm{~km} \mathrm{~s}^{-1} \mathrm{yr}^{-1}$ and a weighted average of blueshifted high-velocity accelerations of $-0.21 \pm 0.08 \mathrm{~km} \mathrm{~s}^{-1} \mathrm{yr}^{-1}$. We note that there is a nonnormal distribution with respect to acceleration systematics due presumably to disk structure. We estimated the azimuth angle, $\phi$, of maser components from the disk midline for a flat-disk model in terms of measurable quantities: the LOS $v_{\text {los }}$ with respect to $v_{\text {sys }}$ and LOS acceleration $\dot{v}_{\text {los }}$, adopting $\phi \approx \sin \phi / \cos ^{4} \phi=G M_{\mathrm{BH}} \dot{v}_{\text {los }} / v_{\text {los }}^{4}$ (Bragg et al. 2000), where $G$ is the gravitational constant, $M_{\mathrm{BH}}$ is the central mass, and $\phi$ is measured from the midline for redshifted high-velocity emission, increasing in the sense of disk rotation. For redshifted high-velocity emission, we found that azimuth angles lie in the range $-8.9^{\circ}$ to $+12.6^{\circ}$ and have a mean and standard deviation of $0.2^{\circ} \pm 3.6^{\circ}$, i.e., are centered on the midline. For blueshifted high-velocity components we determined that azimuth angles lie in the range $166.8^{\circ}$ to $180.5^{\circ}$ and have a mean value of $176.7^{\circ} \pm 6.9^{\circ}$. Since for a warped disk $\phi \propto \sin ^{3} i$, there is a $0.2 \%-2 \%$ error in these values (Herrnstein et al. 2005). Using a flat, edge-on Keplerian disk model and distance of $7.2 \mathrm{Mpc}$

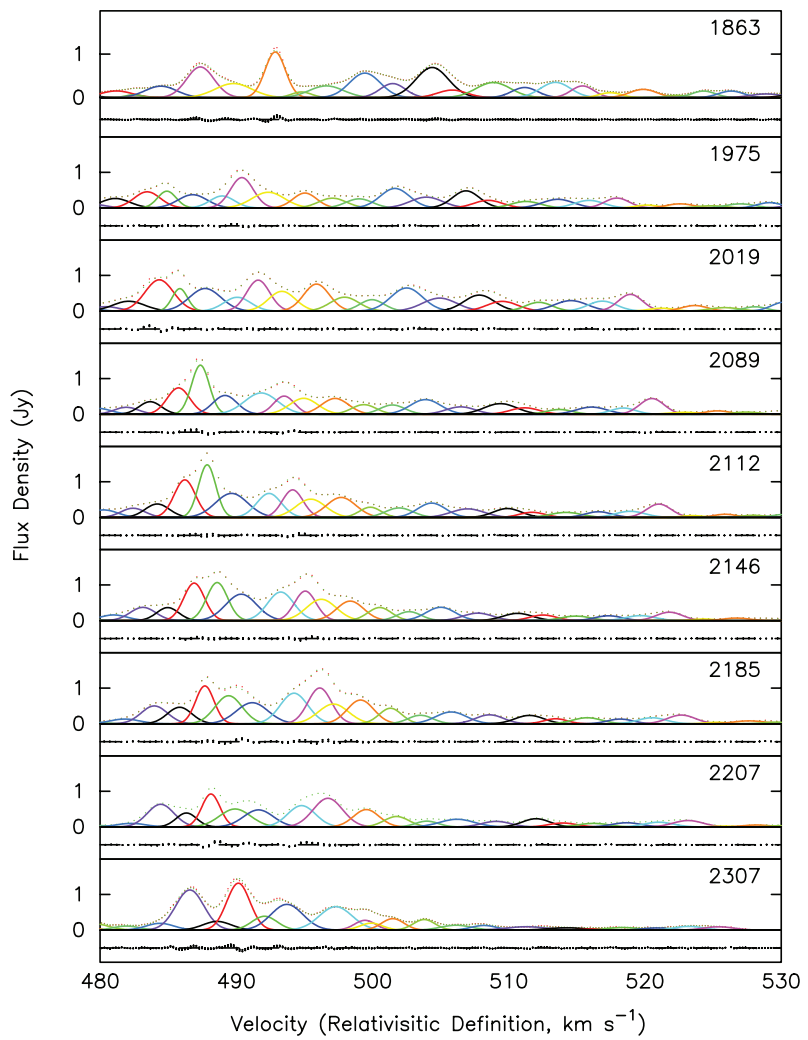

FIG. 5.-Example of Gaussian decomposition for low-velocity emission. A velocity portion of a fit over nine epochs is shown, in which dots are data and black symbols are the residuals, including $1 \sigma$ error bars. Individual model Gaussians are coded using the same color at each epoch, such that Doppler velocity drifts are clearly evident. Numbers in the top right corner of each panel are the number of days since 1994 April 19.

(Herrnstein et al. 1999), we derived disk radii of high-velocity emission in the range $0.17-0.29 \mathrm{pc}$. We measured line widths in the range $1.0-5.0 \mathrm{~km} \mathrm{~s}^{-1}$ for the high-velocity emission.

In Paper I we reported the discovery of new redshifted highvelocity emission at $1562 \mathrm{~km} \mathrm{~s}^{-1}$ and also the detection of emission at $1652 \mathrm{~km} \mathrm{~s}^{-1}$ previously discovered at the GBT by Modjaz et al. (2005). We were unable to estimate the accelerations of these components due to blending and the limited data available.

\subsection{Low-Velocity Emission}

We measured accelerations for four time-consecutive data sets consisting of nine epochs each. We found a significant, reproducible trend in acceleration as a function of component Doppler velocity for each data subset with a systematic variation of $1 \mathrm{~km} \mathrm{~s}^{-1} \mathrm{yr}^{-1}$ across the low-velocity emission range of $\sim 430$ to $550 \mathrm{~km} \mathrm{~s}^{-1}$.

We binned the data from all four fits to yield measured accelerations in the range 7.7 to $8.9 \mathrm{~km} \mathrm{~s}^{-1} \mathrm{yr}^{-1}$ for 55 low-velocity components (Fig. 6; Table 4). The acceleration trend may be associated with a systematic change in the radius at which maximum velocity coherence is achieved due to the disk warp (see $\S 7$ ). We performed a weighted fit to accelerations as a function of velocity and computed a position-velocity (PV) diagram that would be consistent from $v_{\text {los }}=\left(G M / r^{3}\right)^{1 / 2} b$, where $b$ is the impact parameter and $a_{\text {los }}=\left(G M / r^{2}\right) \sin \phi$ for $\phi=270^{\circ}$ and assuming circular rotation. The predicted and observed PV diagrams agree to within $1 \sigma$ for velocities from 390 to $540 \mathrm{~km} \mathrm{~s}^{-1}$ (Fig. 7). For the disk shape of Herrnstein et al. (2005) we find the maser 


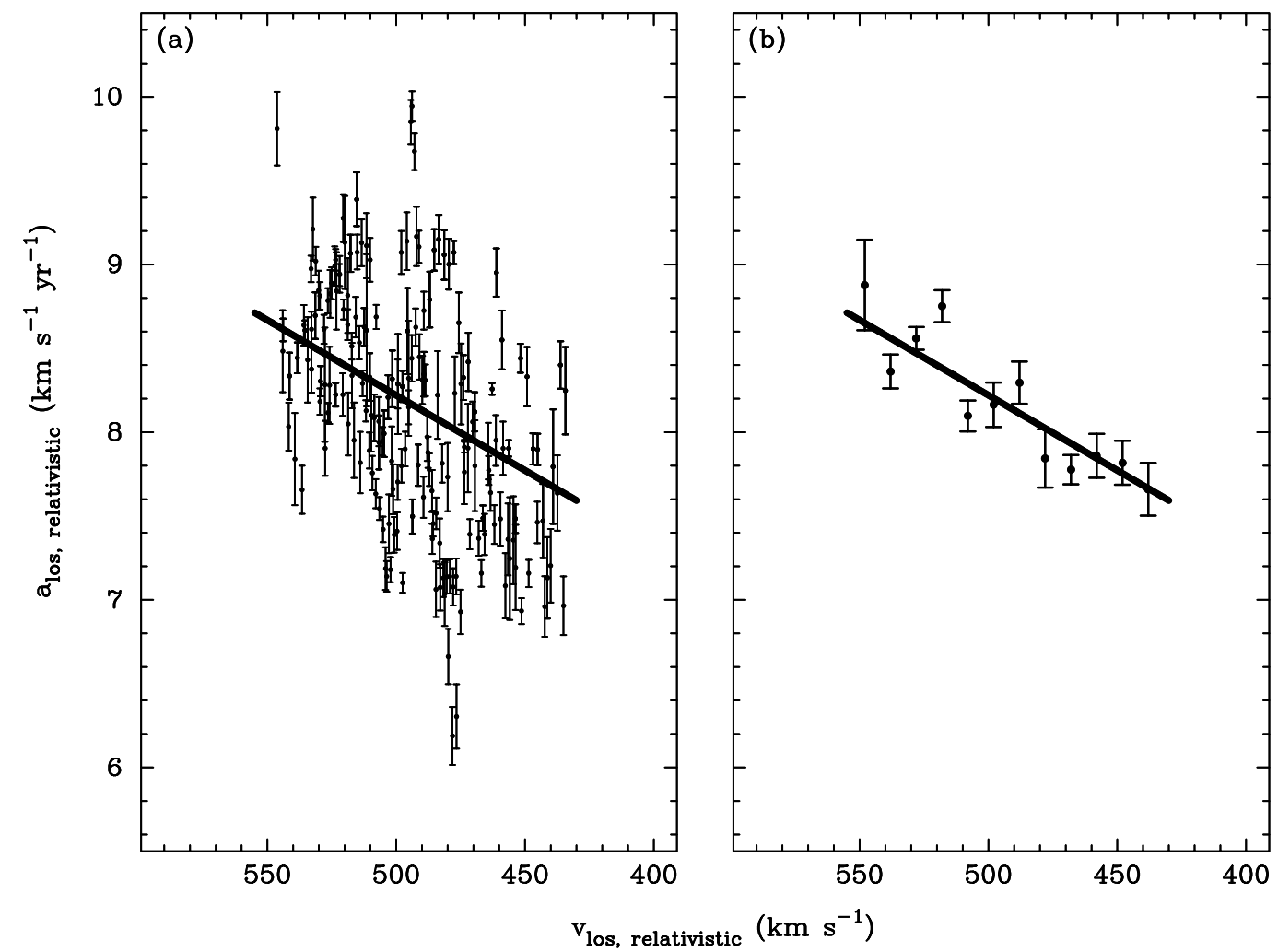

FIG. 6.- Results of Gaussian decomposition for low-velocity emission. (a) Measured accelerations from each of the four time-consecutive nine-epoch fits. Each fit required $\sim 55$ Gaussian components. The line of best fit to all the data is shown. (b) Data in $(a)$ binned into $10 \mathrm{~km} \mathrm{~s}^{-1}$ intervals. The error bars are the standard deviation of the mean in each bin.

components lie in a range of deprojected disk radii of 3.9 to 4.2 mas, as shown in Figure 11 ( 0.14 to $0.15 \mathrm{pc}$ at a distance of $7.2 \mathrm{Mpc}$ ), i.e., $<10 \%$ of the radial extent of observed high-velocity emission. We discuss possible physical origins for the trend in $\S 7$.

\section{COMPARISON WITH PREVIOUS WORK}

Over a monitoring period of $10 \mathrm{yr}$, a high-velocity component orbiting with a period of $700 \mathrm{yr}$ travels $5^{\circ}$ in disk azimuth angle, almost entirely along the LOS. In theory, maser components

TABLE 4

Accelerations for Low-Velocity Maser Doppler Components

\begin{tabular}{|c|c|c|}
\hline $\begin{array}{l}\text { Velocity }^{\mathrm{a}} \\
\left(\mathrm{km} \mathrm{s}^{-1}\right)\end{array}$ & $\begin{array}{l}\text { Acceleration } \\
\left(\mathrm{km} \mathrm{s}^{-1} \mathrm{yr}^{-1}\right)\end{array}$ & $\begin{array}{c}\text { Radius in Geometric Model }{ }^{\mathrm{c}} \\
\text { (mas) }\end{array}$ \\
\hline 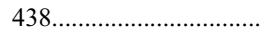 & $7.65 \pm 0.15$ & $4.20 \pm 0.04$ \\
\hline 448 & $7.81 \pm 0.13$ & $4.16 \pm 0.03$ \\
\hline $458 \ldots \ldots \ldots \ldots \ldots \ldots \ldots \ldots \ldots \ldots \ldots \ldots \ldots \ldots$ & $7.85 \pm 0.13$ & $4.15 \pm 0.03$ \\
\hline 468 & $7.77 \pm 0.08$ & $4.17 \pm 0.02$ \\
\hline 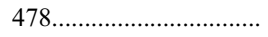 & $7.84 \pm 0.17$ & $4.15 \pm 0.04$ \\
\hline 488 & $8.29 \pm 0.12$ & $4.04 \pm 0.03$ \\
\hline 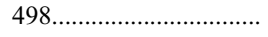 & $8.16 \pm 0.13$ & $4.07 \pm 0.03$ \\
\hline 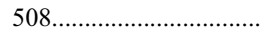 & $8.09 \pm 0.09$ & $4.09 \pm 0.02$ \\
\hline 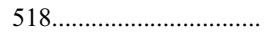 & $8.75 \pm 0.09$ & $3.93 \pm 0.02$ \\
\hline $528 \ldots \ldots \ldots \ldots \ldots \ldots \ldots$ & $8.55 \pm 0.06$ & $3.97 \pm 0.02$ \\
\hline 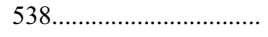 & $8.36 \pm 0.10$ & $4.02 \pm 0.02$ \\
\hline 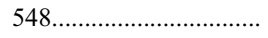 & $8.87 \pm 0.27$ & $3.89 \pm 0.05$ \\
\hline
\end{tabular}

${ }^{\text {a }}$ Data have been binned into $10 \mathrm{~km} \mathrm{~s}^{-1}$ intervals.

b Uncertainties are the standard deviation of the mean for each bin.

c For the best-fitting disk geometry of Herrnstein et al. (2005). See also Fig. 11. could therefore cross the midline during our monitoring, or during the time gaps among studies, and undergo a change in the sign of acceleration. However, a comparison of the different acceleration studies (Table 5) shows that they are in broad agreement. In particular, we compare our acceleration measurements for highvelocity emission with those of Bragg et al. (2000) and Yamauchi et al. (2005) (see Fig. 8). The main difference between our results and those of previous studies is that we measure accelerations for

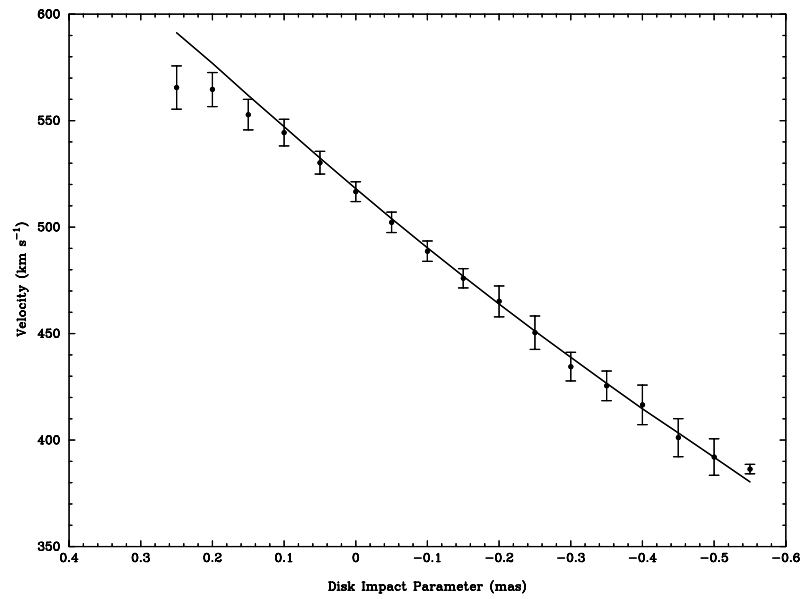

FIG. 7.-Predicted PV diagram for a linear fit to the low-velocity acceleration data (see Fig. 6). Symbols mark observed data from the 18 VLBI epochs of Paper I, binned into 0.05 mas intervals. The curve shows the diagram predicted from the line of best fit to the acceleration data. Curve and data are in close correspondence except at the three most positive values of impact parameter. 


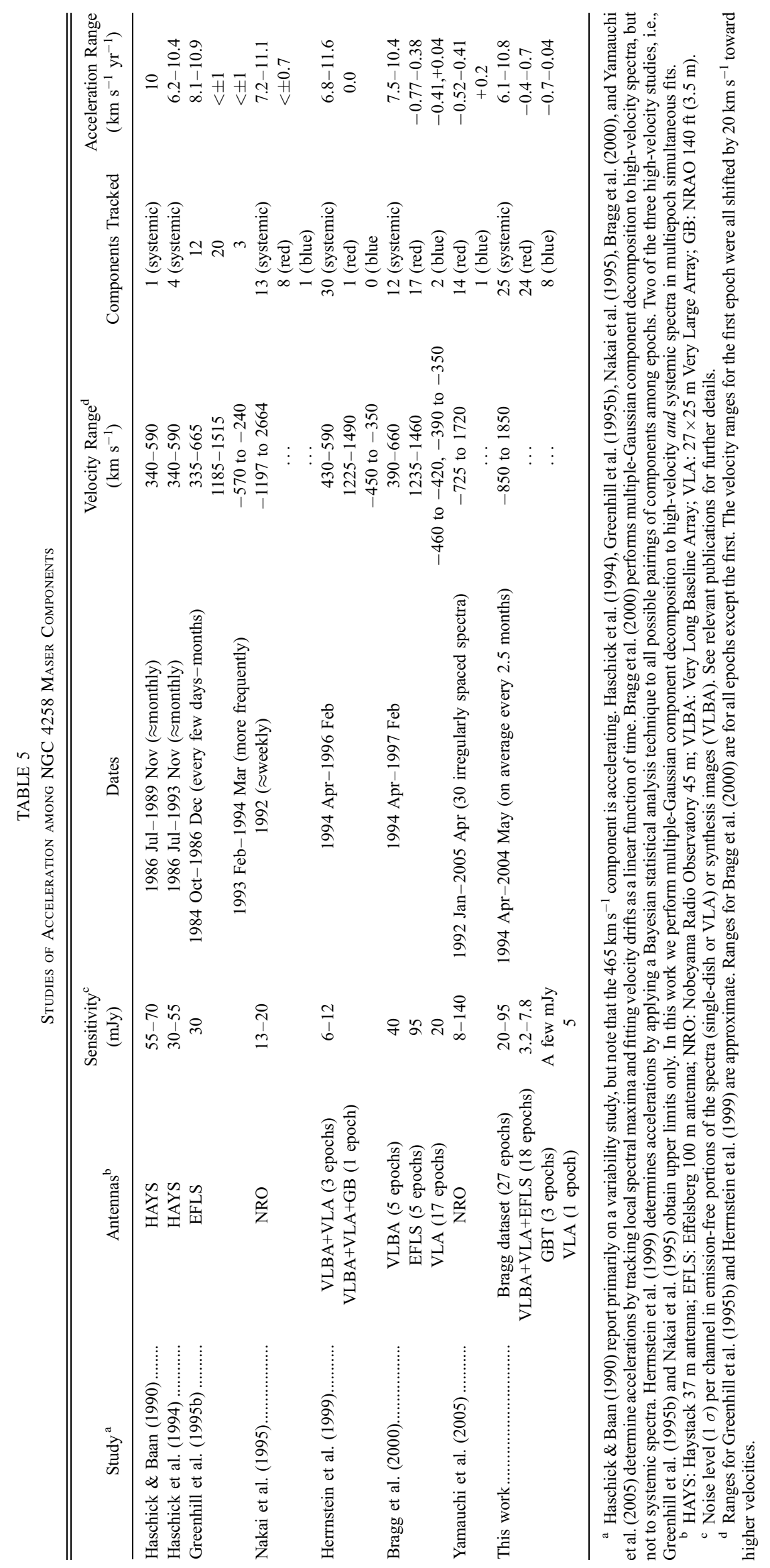




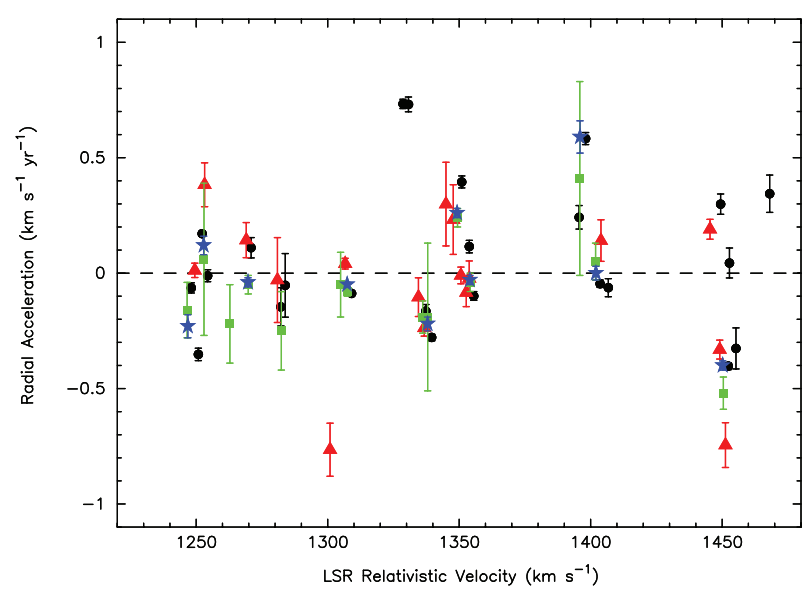

FIG. 8.-Comparison of acceleration measurements for redshifted maser spectral components: the current work (black circles); Bragg et al. (2000) (red triangles); Yamauchi et al. (2005; 1997-2005) (green squares); Yamauchi et al. (2005; 1992-2005) (blue stars). Yamauchi et al. (2005) derive accelerations from their data set over two time ranges, one of which is a subset of the other. All velocities have been converted to a relativistic definition, are referenced to the LSR, and have been adjusted to a common reference date (our monitoring day 2000). Measurements from the different studies are in broad agreement; however, this work tends to identify more components in any given blend compared with earlier work

more components, due to the greater sensitivity and spectral resolution of our data and due to the greater accuracy of the technique we use, to obtain more complete spectrum decomposition.

High-sensitivity and resolution are of particular importance with respect to blueshifted high-velocity components. The addition of the VLBI data of Paper I enabled measurement of accelerations for eight blueshifted components, whereas Bragg et al. (2000) measured accelerations for two blueshifted high-velocity components only: $0.043 \pm 0.036 \mathrm{~km} \mathrm{~s}^{-1} \mathrm{yr}^{-1}$ at $-440 \mathrm{~km} \mathrm{~s}^{-1}$ and $-0.406 \pm 0.070 \mathrm{~km} \mathrm{~s}^{-1} \mathrm{yr}^{-1}$ at $-434 \mathrm{~km} \mathrm{~s}^{-1}$. Yamauchi et al. (2005) obtained a single measurement of $0.2 \pm 0.1 \mathrm{~km} \mathrm{~s}^{-1} \mathrm{yr}^{-1}$ for emission at $-287 \mathrm{~km} \mathrm{~s}^{-1}$. We obtain similar accelerations for the components also measured by Bragg et al. (2000). However, we spectrally resolve the $-287 \mathrm{~km} \mathrm{~s}^{-1}$ emission into three components, with accelerations of $-0.47,-0.12$, and $-0.38 \mathrm{~km} \mathrm{~s}^{-1} \mathrm{yr}^{-1}$ (Fig. 4). Fluctuations in blended features may have led Yamauchi et al. (2005) to obtain a small positive apparent drift rate. If we fit the emission at $-287 \mathrm{~km} \mathrm{~s}^{-1}$ as a single Gaussian component, we also obtain a positive acceleration, in agreement with Yamauchi et al. (2005) within the uncertainties.

The centripetal accelerations for low-velocity emission lie in the same range as those measured in previous studies (Haschick et al. 1994; Greenhill et al. 1995b; Nakai et al. 1995; Herrnstein et al. 1999; Bragg et al. 2000), although binned measurements of the present work tend to be lower at any given velocity (see Fig. 9). This could be due to components at larger radii in the disk or could be associated with the more robust measurement technique used here. A trend in accelerations as a function of component Doppler velocity, with the same sign and similar magnitude as that measured here, is also reported by Haschick et al. (1994) and by Greenhill et al. (1995b) and is evident in the acceleration data of Nakai et al. (1995) and Herrnstein et al. (1999). The geometric model of the maser disk by Herrnstein et al. (2005) indicates that it is warped, both in inclination and position angles, the result of which is to place low-velocity masers in a concavity on the front side of the disk (Figs. 10 and 11). The range of accelerations for low-velocity emission determined by this and other studies could

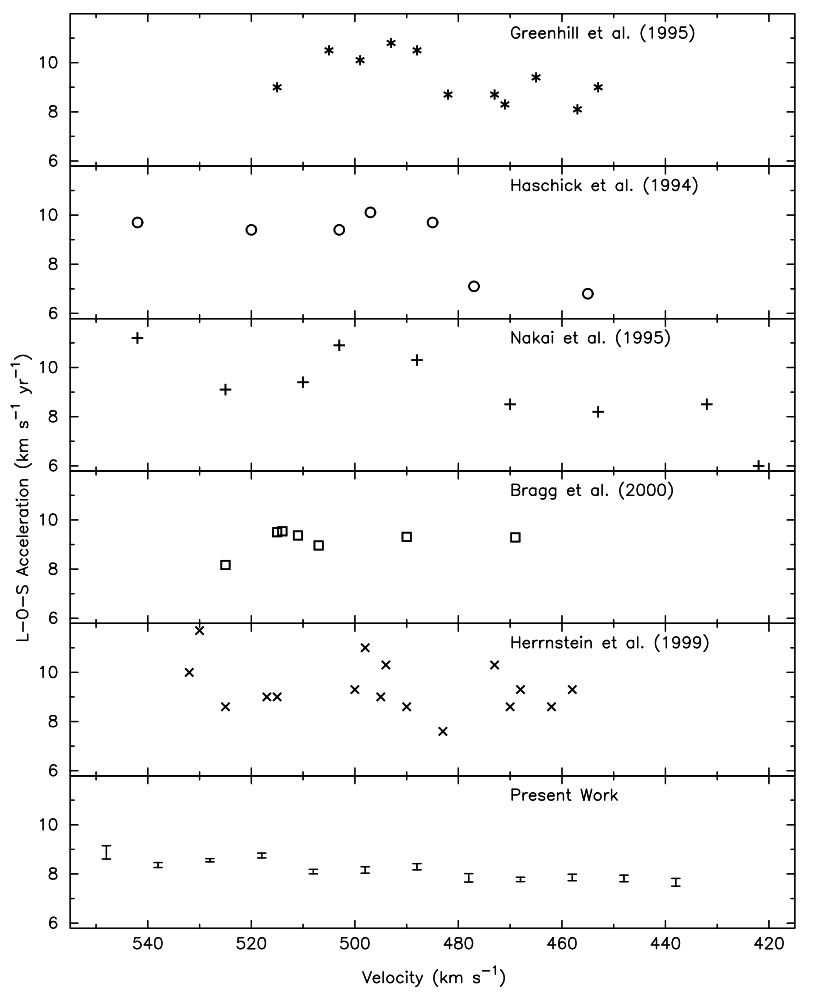

FIG. 9.-Comparison of acceleration measurements for low-velocity maser spectral components. All studies are plotted at the middate of their monitoring period, in order of increasing middate. Only components for which sufficient velocity drift data were obtained have been plotted here. The majority of studies show a trend of increasing acceleration as a function of Doppler velocity. We note that the present work is represented by binned data here, whereas other studies display measurements for individual components. The uncertainties plotted for the binned values are the standard deviation of the mean of each bin, as for Fig. $6 b$. For comparison, in the present work we measure accelerations for $\sim 55$ components in the low-velocity spectrum.

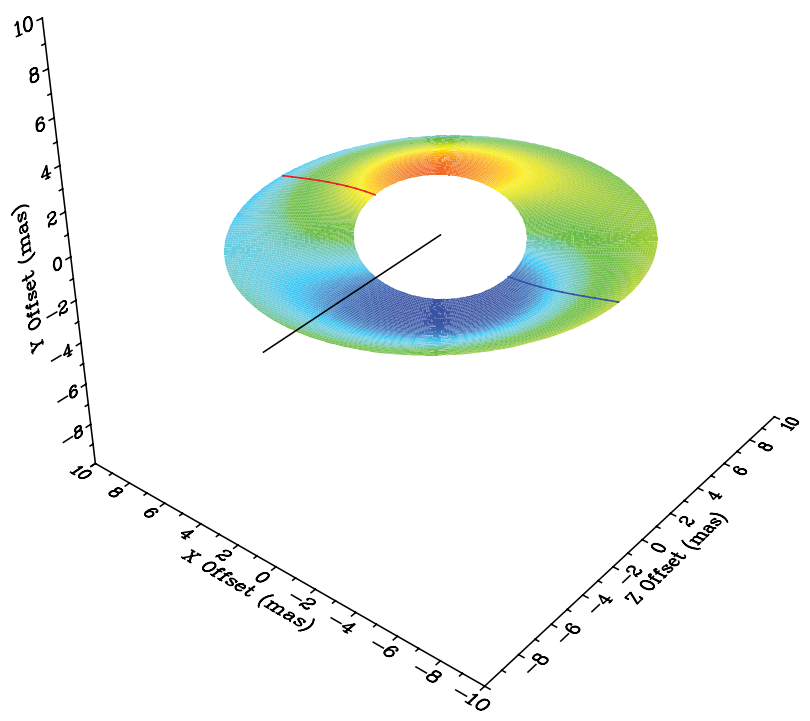

FIG. 10.-Best-fitting maser disk from Herrnstein et al. (2005) viewed from $[-40,40,-50]$. The LOS is shown as a line extending beyond the outer edge of the disk in black along the $z$-direction. Solid color contours show disk elevation, where red is the maximum and dark blue is the minimum. The disk midline for $\phi=0^{\circ}$ and $180^{\circ}$ is shown for redshifted emission (red line) and blueshifted emission (blue line), respectively. Low-velocity (systemic) masers lie in the concavity on the front side of the disk. 


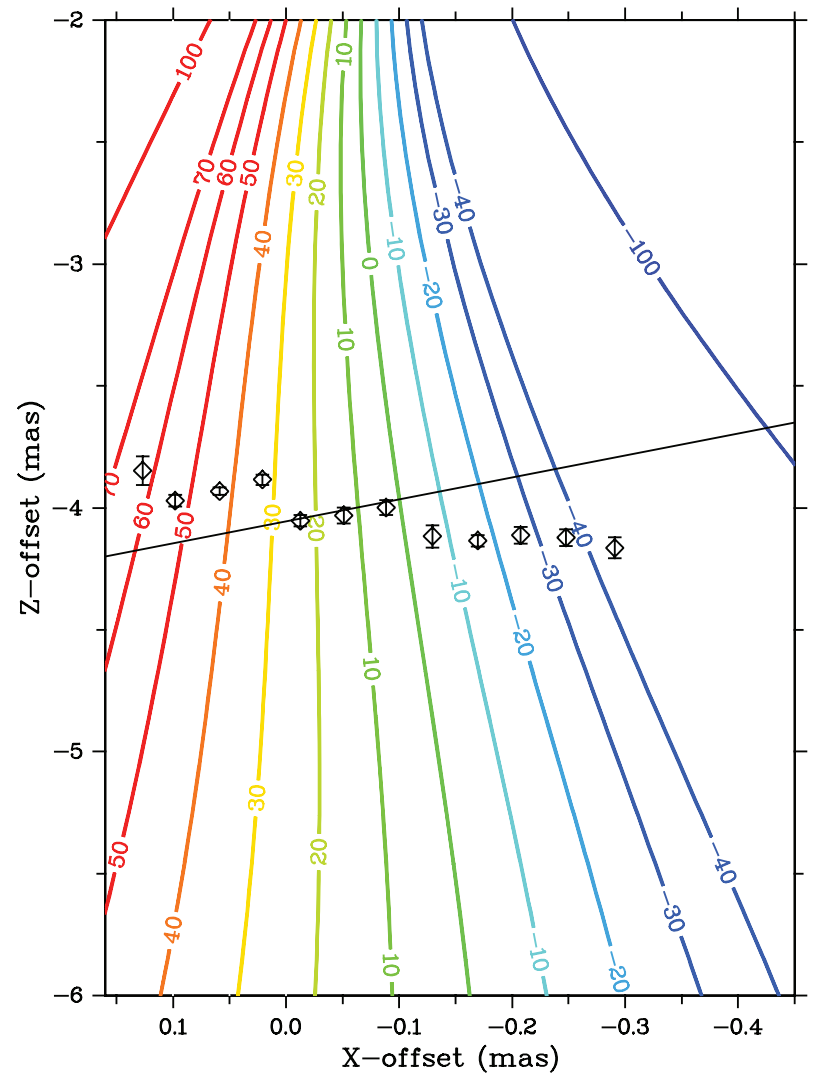

FIG. 11.-Location of the low-velocity emission in the concavity (Herrnstein et al. 2005). Isovelocity contours are marked in color and are in units of $\mathrm{km} \mathrm{s}^{-1}$ relative to $v_{\text {sys }} \mathrm{km} \mathrm{s}^{-1}$ (Herrnstein et al. 2005). The LOS is along the $z$-direction, and therefore a vertical contour would mark a zero velocity gradient in the LOS. The black line marks the bottom of the concavity. The black symbols mark the position of the low-velocity emission, derived from the acceleration data. $\mathrm{Ob}-$ server views along the $z$-axis.

imply a radial spread in low-velocity masers over the relatively broad bottom of the concavity, which is consistent with the model (Herrnstein et al. 2005) and the PV diagram (Fig. 7).

\section{IMPLICATIONS FOR THE ACCRETION DISK FROM HIGH-VELOCITY EMISSION}

\subsection{Disk Geometry}

We can use the accelerations to confirm that the maser dynamic observables reflect real gas dynamics in the NGC 4258 accretion disk. The small accelerations of the high-velocity components suggest that they lie close to the disk midline. For this discussion, it is important to visualize the shape of the disk in the vicinity of the midline, which is in plane of the sky that includes the black hole. In Figure 10 we show the geometry of the maser disk determined by Herrnstein et al. (2005). On the midline the disk shape is defined by the position angle warp, as shown in Figures 12 and 13, respectively, for the red and blue side of the disk. That is, the expected midline curve is $y=r \cos \alpha_{r}$, where $y$ is the projected vertical position of the warped disk on the sky relative to the disk dynamical center in units of milliarcseconds, $\alpha_{r}$ is the disk position angle measured north of east in the plane of the sky (the $x-y$ plane in Fig. 10) given by $\alpha_{r}=65.6^{\circ}+5.04^{\circ} r-0.22^{\circ} r^{2}$ (Herrnstein et al. 2005), and $r$ is the radial position of masers in the disk in milliarcseconds. We take $r \sim b$, where $b$ is the impact parameter in the plane of the sky measured from the disk dynamical center.
Over the radial range of emission from 4 to 8 mas on the red side of the disk, the position angle varies from $84^{\circ}$ to $92^{\circ}$. The "tilt" of the disk at the midline is given by the inclination warp, which is described by $i_{r}=107^{\circ}-2.29^{\circ} r$ (Herrnstein et al. 2005). Hence, for the red side of the disk, $i_{r}$ varies from $97^{\circ}$ at 4 mas to $89^{\circ}$ at 8 mas. The range is the same for the blue side of the disk. It is important to note that at radii of 7.4 mas, we view the disk exactly edge on. Inside this radius, the near side of the disk is tipped down with respect to the observer, while outside it is tipped up. Masers at radii smaller than 7.4 mas, that lie above the midline in VLBI images, should be behind the midline plane in the disk and have negative accelerations. Similarly, masers beyond 7.4 mas radius, which appear above the midline in Figures 12 and 13, are expected to be in front of the midline plane and should have positive accelerations.

We compared the vertical deviations in the VLBI image caused by the disk projection on the sky (see Figs. 12 and 13), $y_{\text {image, }}$ with the vertical deviations expected from the accelerations, $y_{\text {acc }}$. To derive $y_{\text {acc }}$ for each maser, we first computed its distance in the $z$-direction from the midline from its azimuth (which comes from its acceleration), that is, $z=b \tan \phi$, where $\phi$ is the azimuth angle of the maser listed in Tables 2 and 3. From this azimuth offset and the local slope of the warped disk at radius $r, y_{\text {acc }}$ was calculated as the projected offset from the midline. The plot of $y_{\text {acc }}$ versus $y_{\text {image }}$ is shown in Figure 14. The errors in $y_{\text {image }}$ are the quadrature sum of the errors in the VLBI position and the errors in the midline definition. The errors in $y_{\text {acc }}$ are the result of acceleration measurement errors, inclination model errors, and errors due to a finite vertical distribution of masers. We adopt a disk thickness of $12 \mu \mathrm{as}$, the value determined from low-velocity emission in Paper I. The disk thickness corresponds to a temperature of about $600 \mathrm{~K}$ for hydrostatic equilibrium. In order to make the reduced $\chi^{2}$ of the fit equal 1 , it was necessary to add a systematic rms acceleration of $0.1 \mathrm{~km} \mathrm{~s}^{-1} \mathrm{yr}^{-1}$. Since the magnitude of the maser accelerations is up to $\sim 10 \mathrm{~km} \mathrm{~s}^{-1} \mathrm{yr}^{-1}$, the systematic errors in the acceleration measurements are at the 1\% level. The errors in Figure 14 reflect these contributions. Note that there is one significantly deviant point at $2.8 \sigma$ (the one at $1271 \mathrm{~km} \mathrm{~s}^{-1}$ and $r=7.37$ mas).

\subsection{Spiral Structure}

Accelerations and positions of high-velocity emission can also be used to investigate theories of spiral structure in the accretion disk of NGC 4258. First, Maoz (1995) noted periodic clustering of high velocity component radii in a visual analysis of the single VLBI map by Miyoshi et al. (1995). Maoz inferred a characteristic wavelength of $\lambda_{\text {char }}=0.75$ mas $(0.027 \mathrm{pc})$ for the clustering and attributed it to spiral density waves that form by swing amplification. Spiral density waves can form in a disk that is unstable to nonaxisymmetric perturbation in the $1<Q \leq 2$ regime, where $Q$ is the Toomre stability parameter (Toomre 1964). "Swing" refers to the fact that a nonaxisymmetric disturbance is converted to a trailing spiral arm (in which the outer tip points backward, opposite the direction of disk rotation) due to shearing by differential rotation. "Amplification" occurs during this process since the direction of the shear flow of the arm is the same direction as local epicyclic motions, which enhances the self-gravity of the arms. To satisfy this stability regime, Maoz (1995) required densities in spiral arms of $n\left(\mathrm{H}_{2}\right)=10^{8}-10^{10} \mathrm{~cm}^{-3}$, which are sufficient to produce $22 \mathrm{GHz}$ maser emission (e.g., Cooke \& Elitzur 1985), while those in the interarm regions are not. In the theory of Maoz (1995) masers are discrete physical clumps that can move freely through different spiral arms and can be tracked between epochs.

Maoz \& McKee (1998, hereafter MM98) also propose that maser emission could arise behind spiral shocks in the NGC 4258 


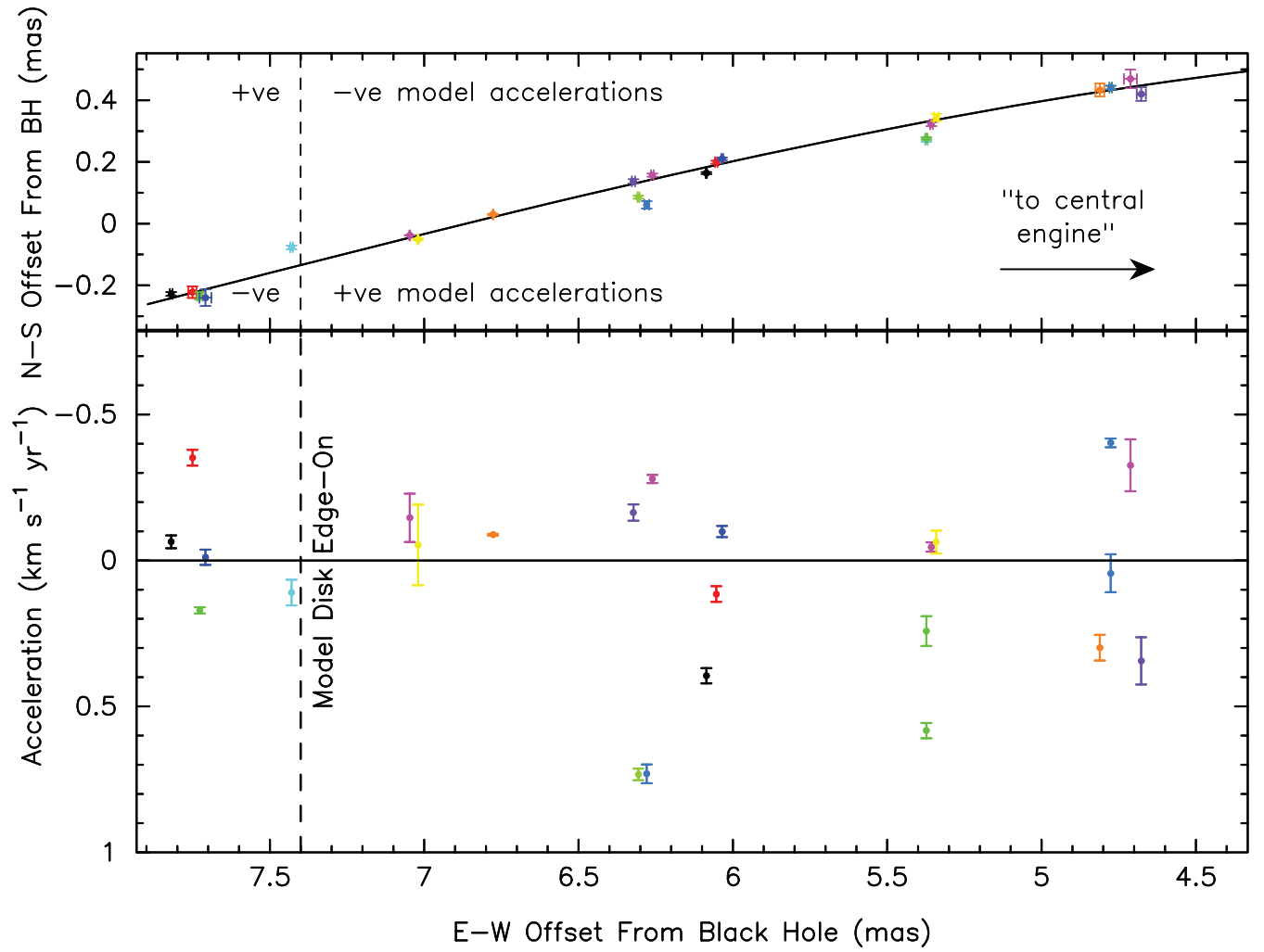

FIG. 12.-Sky positions and accelerations of fitted redshifted maser components. Bottom: LOS accelerations plotted as a function of disk east-west offset. The solid line marks zero LOS acceleration, which should occur when masers are on the disk midline. Top: Sky positions of the corresponding maser components. The solid line indicates the disk midline predicted by the geometric model of Herrnstein et al. (2005). Note that, for this model, masers above the model midline (top) should also have negative accelerations at radii $<7.4$ mas and positive accelerations at greater radii.

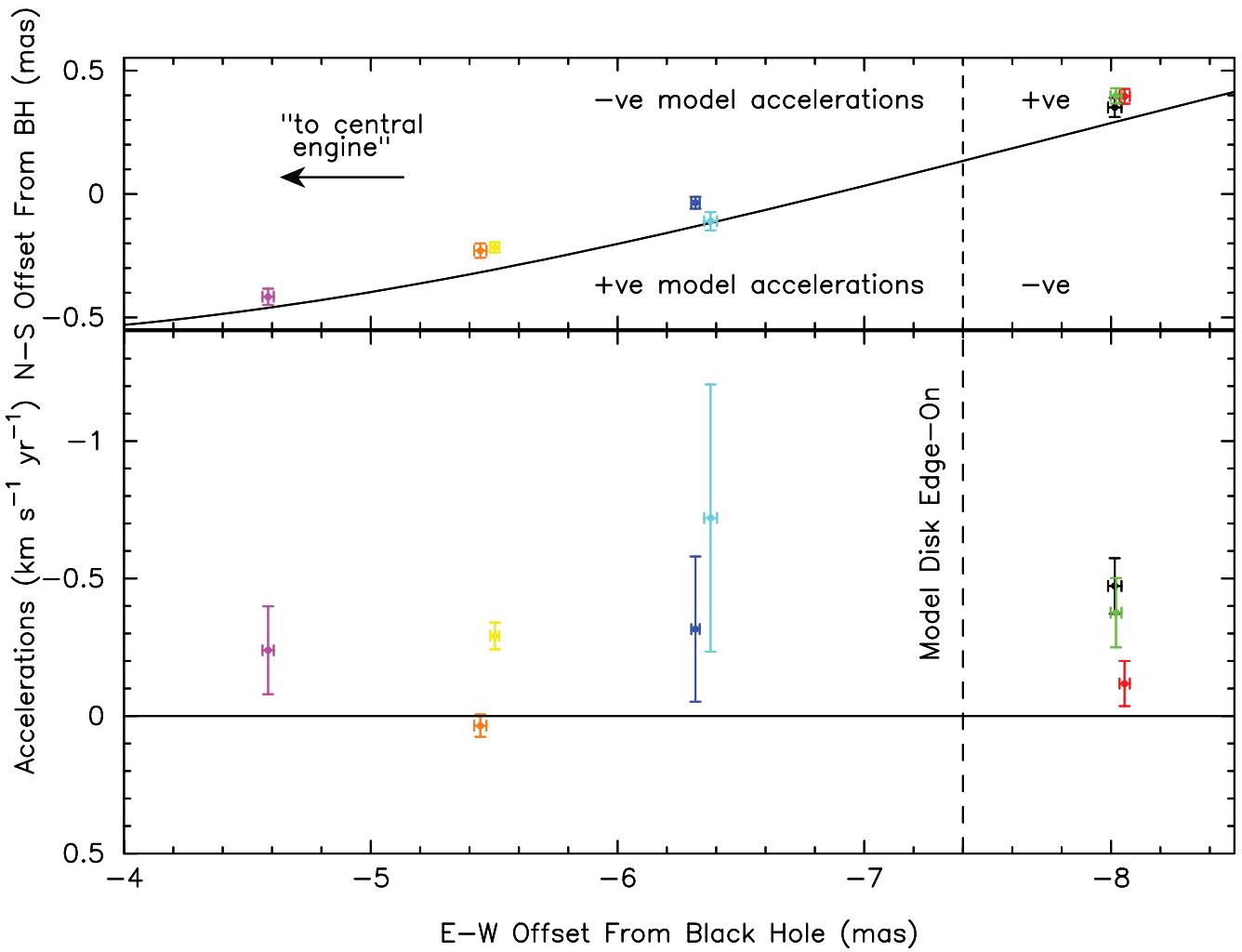

FIG. 13.- Same as Fig. 12, but for fitted blueshifted high-velocity components. 


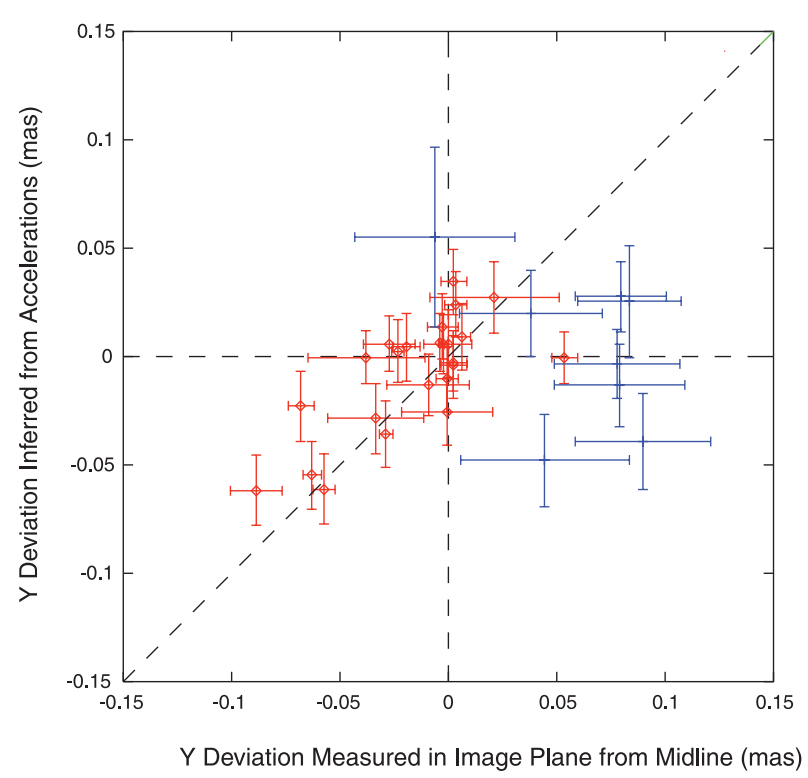

FIG. 14.- Vertical deviations from the warped disk of Herrnstein et al. (2005) calculated using acceleration data and VLBI data. See $\S 6.1$ for details.

disk, based on density and velocity coherence arguments. The shocks occur at the interface of spiral arms sweeping through the disk. In this case masers may not be discrete entities but a wave phenomenon, with emission arising at the tangents of the spiral shocks along the LOS, where the path length for amplification is highest. At different epochs, maser emission would not originate from the same gas condensations. The accelerations of the masers would no longer reflect pure Keplerian dynamics, as the action of the shock causes the excitation points for masers to move to greater radii in the disk. All maser velocities would shift to lower absolute values. This process would manifest itself observationally as positive accelerations for blueshifted high-velocity components and as negative accelerations for redshifted highvelocity components. Different spiral theories are schematically represented in Figure 15.

\subsubsection{Spiral Density Wave Model (Maoz 1995)}

Maoz (1995) argues that (1) maser components are periodic in disk radius with a characteristic wavelength of $\lambda_{\text {char }} \sim 0.75$ mas and (2) are located within several degrees of the disk midline. Point 2 is required to avoid a wide range of accelerations of the masers due to spiral arms, which are not observed. If masers are near the midline, then the noncircular ( peculiar) motion is largely perpendicular to the arms (Toomre 1981) and perpendicular to the LOS (and therefore undetectable in Doppler shifts). Maoz (1995) estimates that the component of the peculiar motion along the LOS for high-velocity emission would not exceed $4.5 \mathrm{~km} \mathrm{~s}^{-1}$, comparable to the measured accuracy of the Keplerian rotation curve (Maoz 1995).

An apparent periodicity in the distribution of maser sky positions is obvious from VLBI images of the data. For formal quantification, we performed an analysis using the Lomb-Scargle periodogram for the 18 epochs of VLBI data described in Paper I. First, we created a "binary" data set from the VLBI positions, in which any disk position that had detected emission was assigned a "flux density" of 1 , to ensure that disparity in flux density between red- and blueshifted emission did not affect the outcome of the analysis. We gridded the binary data set at intervals of 0.005 mas, i.e., on scales much shorter than any expected
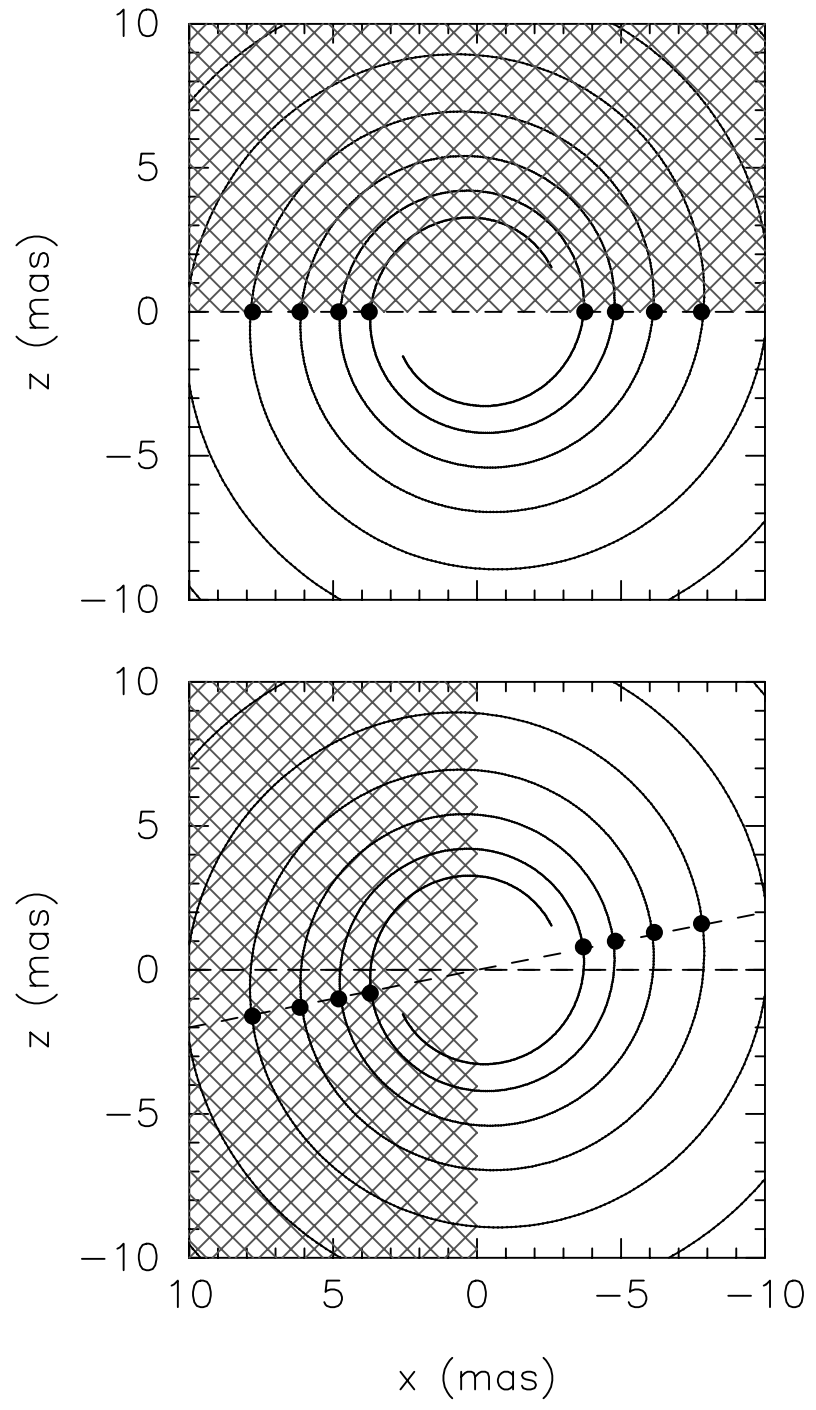

FIG. 15.-Comparison of spiral structure model predictions in the NGC 4258 accretion disk. Top: Schematic of maser disk according Maoz (1995). Masers ( filled circles) are periodically located in density peaks of trailing spiral structures (represented here by a two-arm logarithmic spiral). Accelerations are negative behind the midline (cross-hatched area) and positive in front of the midline. Components should occur within several degrees of the midline for maximum velocity coherent maser path lengths. Bottom: Schematic of the maser disk according to MM98. Masers ( filled circles) are periodically located in the disk, but offset from the midline by $2^{\circ}$ (note the angle is exaggerated here). Redshifted highvelocity components have negative accelerations (cross-hatched area), whereas blueshifted component accelerations should be positive, statistically speaking. Maser emission occurs at the longest velocity coherent path lengths along the LOS through spiral arms.

$\lambda_{\text {char }}$ emission, and placed zeroes at positions at which we did not detect any in the VLBI maps. Using the periodogram, we found a dominant period in the binary distribution of high-velocity maser positions of 0.75 mas (Fig. 16), at the same value as that estimated by Maoz (1995). We also predicted that emission should exist at a velocity of about $-330 \mathrm{~km} \mathrm{~s}^{-1}$ in the blueshifted spectrum. In subsequent observations obtained with the GBT on 2003 October 23 , the emission predicted by the periodogram analysis was detected at $-329 \mathrm{~km} \mathrm{~s}^{-1}$ (Fig. 17). At $<10 \mathrm{mJy}$, this emission would not have been detected in our VLBI observations.

We note that the model of Maoz (1995) has densities in the spiral arms that can produce maser emission but not in the interarm 


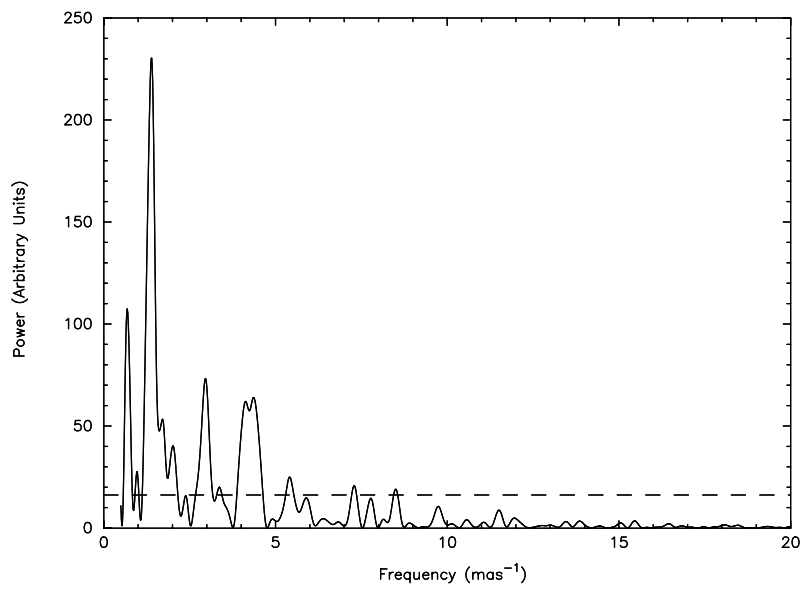

FIG. 16.-Periodogram of the positions of the high-velocity components in the NGC 4258 disk. The dominant frequency peak at 1.33 mas $^{-1}$ corresponds to a characteristic wavelength of 0.75 mas. The dashed horizontal black line marks the $3 \sigma$ significance level. We confined our analysis to "wavelengths" of $<2$ mas, since longer periods would not be well constrained by our data set.

regions. In the denser spiral arms, gas condensations naturally form. In this case, discrete maser-emitting condensations could be tracked as they move across an arm. They are not necessarily persistent in the interarm region, i.e., discrete maser condensations may only exist within arms, and disperse in the interarm regions. As a result, clumps responsible for low-velocity emission do not survive to beam high-velocity emission toward the observer after one-quarter of a rotation, and, conversely, high-velocity clumps do not survive to eventually be seen as low-velocity clumps.

\subsubsection{Spiral Shock Model (MM98)}

The specific predictions of MM98 for masers, in addition to periodicity in maser sky positions, are that (1) blueshifted components are accelerating and they are located behind the disk midline and (2) redshifted components are decelerating and should be located in front of the midline. By contrast, in a disk dominated by Keplerian dynamics, masers in front of the midline should have a positive sign of acceleration and masers located behind the midline should have a negative sign of acceleration.

We do not find that the accelerations of high-velocity components conform to the MM98 model. Both the red-and blueshifted component accelerations are statistically near zero (see $\S 4.1$ and Tables 2 and 3), although there is a $2.6 \sigma$ bias of the latter to negative accelerations, which is not in agreement with predictions of MM98. We note that Yamauchi et al. (2005) presented evidence in support of MM98. However, Yamauchi et al. (2005) measured the acceleration of one blueshifted component only, which had a positive acceleration at the $2 \sigma$ significance level. We obtain a negative acceleration for the same component (see $\S 5$ ) when it is decomposed into three features. Of the eight blueshifted components for which we measured accelerations, only one is positive. Bragg et al. (2000) also do not find accelerations that would support the MM98 theory.

\subsubsection{Other Origins for the Periodicity}

We also considered the possibility that orbiting objects (e.g., stars) create the substructure in the NGC 4258 accretion disk, with some resonance mechanism, for example, causing regularity in the spacings. If the stellar density is the same in the central regions of NGC 4258 as it is in the center of the Milky Way, then integrating $\rho_{*}(R)=1.2 \times 10^{6}(R / 0.425)^{-\alpha}$ between $R=0.14$ and
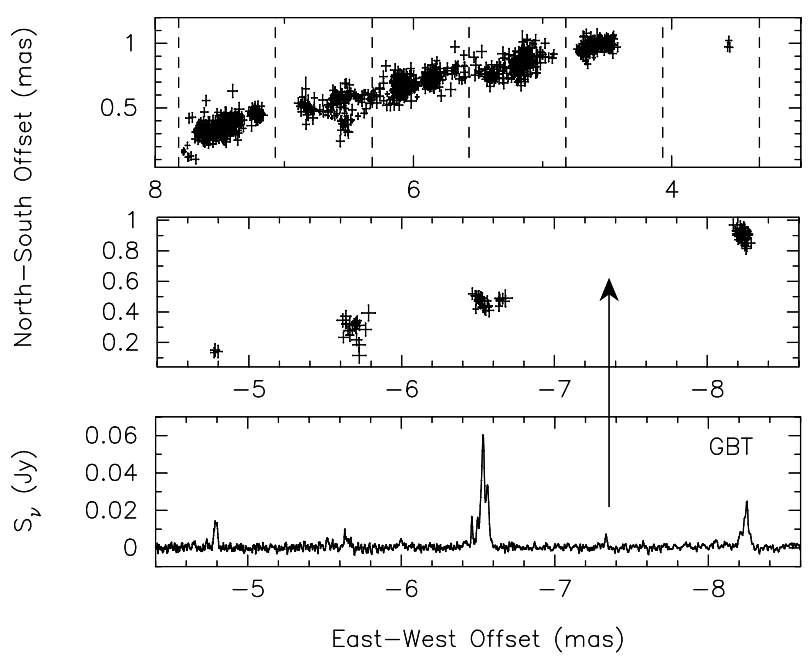

FIG. 17.-Top: Sky positions of redshifted high-velocity emission in the NGC 4258 disk obtained using VLBI over 18 epochs. Dashed lines mark nodes at characteristic wavelengths of 0.75 mas, proposed by Maoz (1995). Middle: Blueshifted components in the NGC 4258 disk. Bottom: GBT spectrum of the blueshifted high-velocity emission taken on 2003 October 23 . Velocity has been represented as approximate east-west offset. The arrow marks the velocity of the "missing" blue component predicted by the Fourier analysis and detected independently in the spectrum.

$0.28 \mathrm{pc}$ and assuming $\alpha=1.4$ (from Genzel et al. 2003) yields a stellar mass of $2 \times 10^{4} M_{\odot}$ in the spherical volume containing the maser disk; so there are likely at least several thousand stars. The cumulative effect of many passages of stars around the black hole might bring them into a circular orbit corotating with the disk, although we note that highly eccentric stellar orbits are found in our Galactic center. Each star could then either open up a gap in the disk (if the disk is thinner than the star's Hill radius and the viscosity in the disk is sufficiently low) or accrete material from the disk. The vertical thickness of the maser disk is measured to be $12 \mu$ as (Paper I). We therefore estimate the lower limit on the mass of a star (or stellar cluster) required to create a gap completely through the disk to be $>0.05 M_{\odot}\left[2 r_{\mathrm{Hill}} \approx 2 r_{\mathrm{orb}}\left(m_{*} / 3 M_{\mathrm{BH}}\right)^{1 / 3}=\right.$ $0.0003 \mathrm{pc}$ for $\left.\overline{r_{\mathrm{orb}}}=0.2 \mathrm{pc}\right]$, such that any star, even a brown dwarf, could create a gap in the disk equal to its thickness. We note that a number of massive $\mathrm{He}$ I stars (of mass $30-100 M_{\odot}$ ) exist between 0.04 and $0.5 \mathrm{pc}\left(1^{\prime \prime}\right.$ to $\left.12^{\prime \prime}\right)$ of the Galactic center (Genzel et al. 2003) and that a corresponding $\sim 100 M_{\odot}$ star in the NGC 4258 disk would create a gap of $2 r_{\text {Hill/Roche }}=$ $0.006 \mathrm{pc}$. The stars would be at radii several orders of magnitude greater from the SMBH than their tidal disruption radii $r_{\text {tidal }} \simeq$ $r_{*}\left(M_{\mathrm{BH}} / m_{*}\right)^{1 / 3}$, where $r_{*}$ and $m_{*}$ are the radius and mass of the star, respectively (see, e.g., Bogdanović et al. 2004).

\section{IMPLICATIONS FOR THE ACCRETION DISK FROM LOW-VELOCITY EMISSION}

The range of accelerations measured for low-velocity components is consistent with the warped disk model of Herrnstein et al. (2005) (see our Figs. 10 and 11). However, the systematic trend in component acceleration with Doppler velocity (see $\S$ 4.2 ) is not predicted by this model. The trend is likely connected to some aspect of the warped disk geometry that results in a preferred locus in disk radius and azimuth angle within which lowvelocity maser action is favored (Fig. 18a) as a result of geometry and orientation to the LOS, and we investigate this point further in the next paper of this series, in which we report new modeling of the 3D disk structure and dynamics based on the expanded 


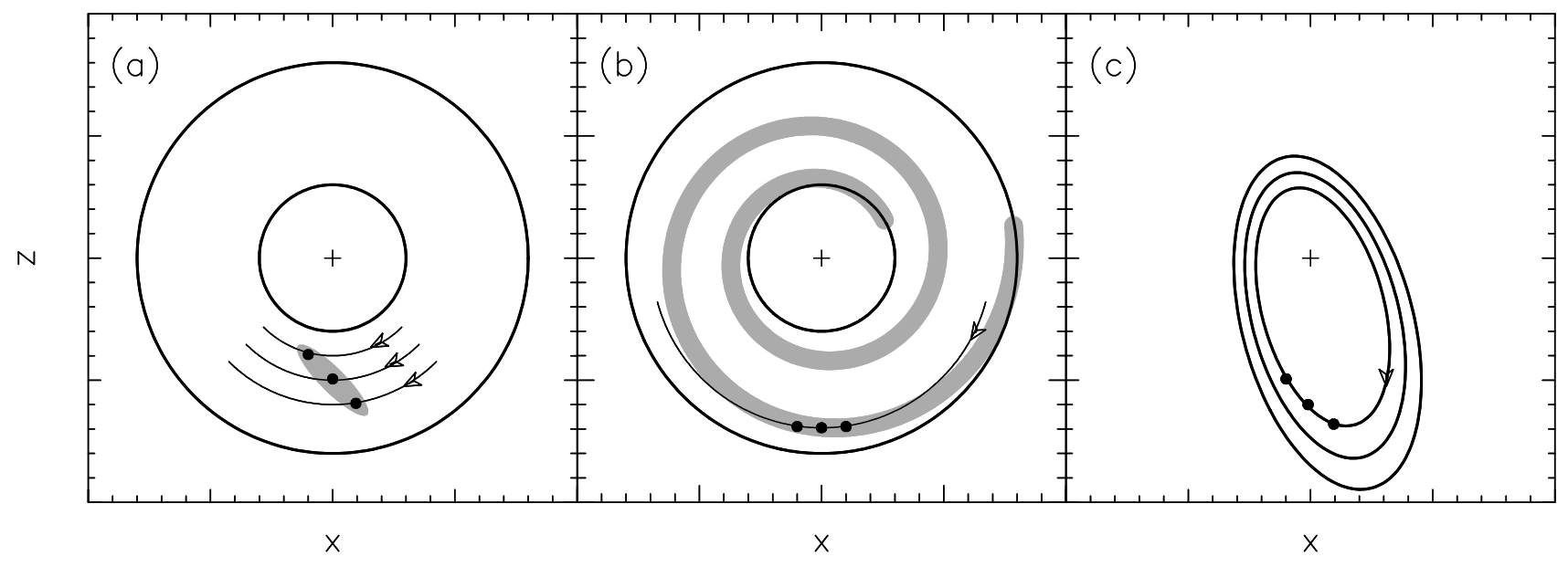

FIG. 18. - Scenarios for producing the trend in LOS accelerations of low-velocity emission as a function of component Doppler velocity. The LOS direction is along the $z$-axis. (a) Masers in circular, Keplerian orbits with locations determined by disk geometry and orientation. In this scenario components ( filled black circles) are in circular orbits about the black hole (black cross) but only produce visible maser action within a favorable locus of disk radius and azimuth angle (shown in gray) determined by the warping of the disk and tangency of the LOS to the disk. The acceleration trend in this case is due to emission from a number of different maser components in orbits of different radii. (b) Masers in a circular, Keplerian orbit that crosses a spiral arm. In this scenario maser components are in a circular orbit about the black hole and encounter a spiral arm that perturbs the orbital motions (the arm is represented here by a gray logarithmic spiral, but a more realistic scenario would be fragmentary, as in flocculent spiral galaxy disks.) The acceleration trend in this case is due to the passage of maser components through a spiral arm of lower pattern speed than the local Keplerian velocity (see Fig. 19). (c) Masers in noncircular, confocal, Keplerian orbits. Maser components in the same elliptical orbit by definition are at different radii and exhibit different accelerations, but they may instead lie on different orbits with the same effect.

data set presented in Paper I and in this paper. In order to understand other types of "higher order" phenomena that could give rise to the trend, however, we attempted to reproduce the observations by (1) assuming circular orbits in the disk with a spiral arm in the low-velocity maser region (Fig. 18b) and (2) allowing for eccentricity in maser orbits (Fig. 18c). In the preliminary investigations that follow, we have assumed that the maser disk is flat and is viewed edge-on.

\subsection{Spiral Structure}

In order to investigate whether spiral structure could give rise to the gradient evident in accelerations of low-velocity components with Doppler velocity, we performed $N$-body simulations for a maser orbiting a supermassive black hole $\left(3.8 \times 10^{7} \mathrm{M}_{\odot}\right.$; Herrnstein et al. 2005) in a flat disk and encountering a spiral arm of various masses (Fig. 19). For the simulations, we used a direct $N$-body integration code, NBODY0 (Aarseth 1985), in which each particle is followed with its own integration step to account for a range of dynamical rates. See Aarseth (1985) for more details.

We represented a spiral arm at $t=0$ (Fig. 19a) using 8000 particles distributed along an arbitrarily chosen trailing logarithmic spiral arm of $r=r_{0} \exp \left[b\left(\phi-\phi_{0}\right)\right]$, in which $b=0.42, \phi_{0}=0^{\circ}$, and $r_{0}=1.5$ mas or $0.06 \mathrm{pc}$, with a radial extent of $10^{-2} \mathrm{pc}$. The pattern speed of the arm was set to be half that of the Keplerian orbital velocity (by reducing the force on arm particles by a factor of 4) at any given radius, and gravitational interaction between arm particles was ignored. We based values for the fractional radial extent and pattern speed of the arm roughly on those of Galactic spiral arms. To avoid potentially infinite acceleration between arm particles and the maser, and to better represent a smooth distribution of mass in the arm, we included a "softening" term of $5 \times 10^{-3} \mathrm{pc}$ that was added in quadrature to each maserparticle separation.

We varied the mass contained in the arm (bounded by the upper mass limit for the maser disk determined by Herrnstein et al. [2005] of $M_{\text {disk,upper }}=9 \times 10^{5} M_{\odot}$ ) for different runs of the code. For any given run, we followed the evolution of a maser cloud through the spiral arm (Figs. $19 a-19 c$ ) for several hundred years, sampling every $0.55 \mathrm{yr}$.

The computed LOS accelerations for the maser showed significant deviations from that of a massless arm for arm masses greater

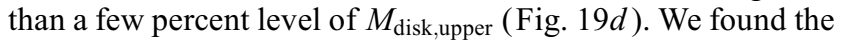
"amplitude" of the acceleration deviation to be directly proportional to arm mass and could reproduce observed trends in acceleration (see $\S 4.2$ ) for an arm mass of $15 \%$ of the upper limit maser disk mass. In the simulations, the acceleration trend is reproduced when the maser is "in" the spiral arm (between 100 and $200 \mathrm{yr}$ in Fig. 19).

We note that our calculations include gravitational effects only and do not attempt to incorporate shocks or magnetohydrodynamic effects of spiral density wave theory.

\subsection{Eccentric Accretion Flows}

While accretion flows are generally believed to circularize on timescales much shorter than the viscous timescale, Statler (2001) showed that elliptical orbits of $e \leq 0.3$ can be long lived if the disk is thin and if the orbits are nested and confocal, with precession rates that maintain the alignment.

We describe a system of nested elliptical orbits of identical eccentricity, $e$, of differing semimajor axes, $a_{\text {semi }}$, and with the same periapsis angle, $\omega$, about a supermassive black hole. The periapsis angle is defined here as the angle between the sky plane to the periastron point on each orbit (increasing from the "redshifted" midline in the sense of disk rotation). In the discussion that follows, it is important to note that no matter the $e$ and $\omega$ of the system, redshifted high-velocity maser emission components must originate from an azimuthal angle of $\phi \approx 0^{\circ}$ (where $\phi-\omega$ is the angle of the component from periastron), and blueshifted components from $\phi \approx 180^{\circ}$ on the basis of velocity coherence and acceleration arguments.

We first used the symmetry of the position-velocity (PV) diagram of high-velocity emission to place limits on eccentricity. At any given radius in the disk in the plane of the sky (i.e., where the 

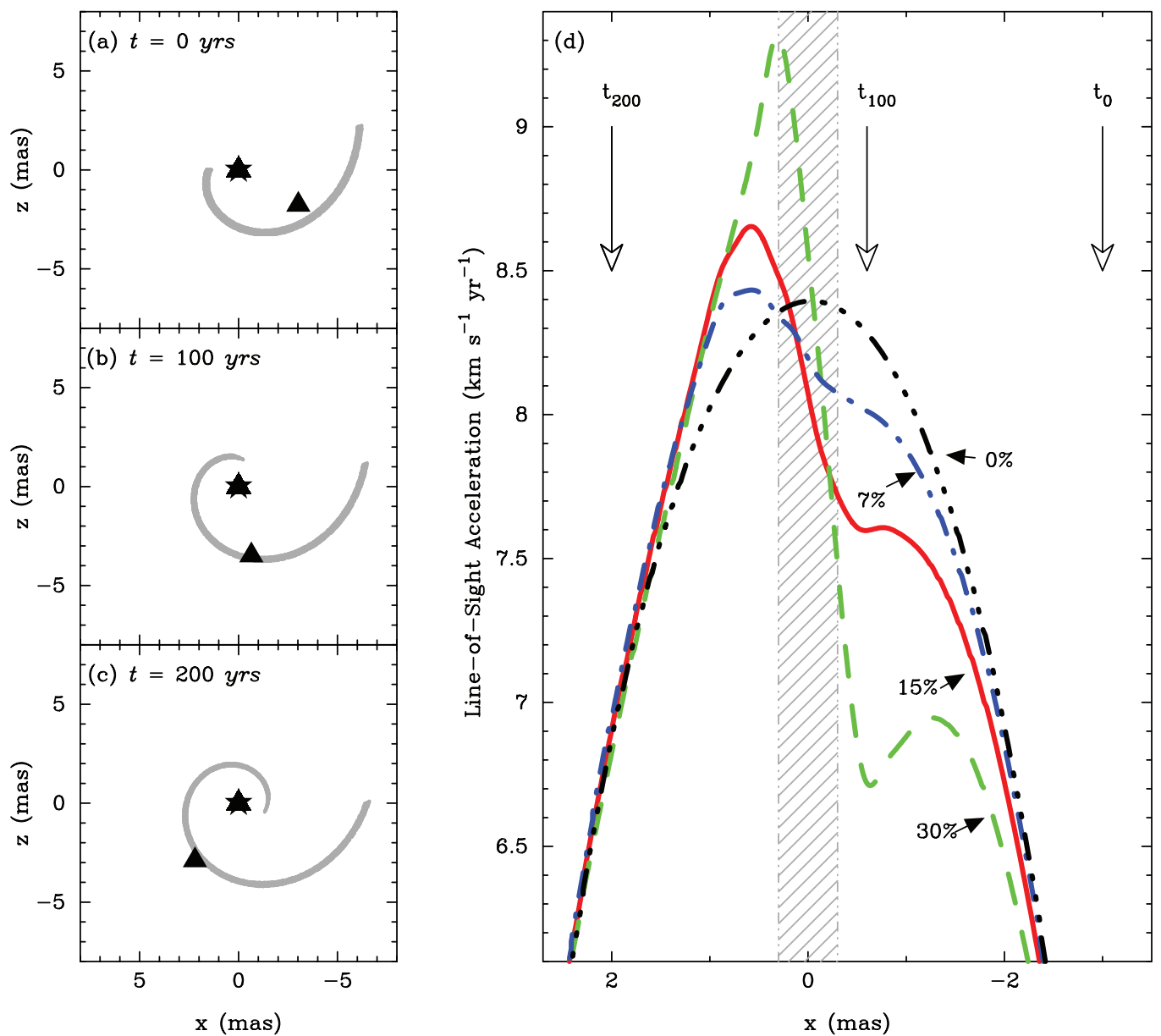

FIG. 19.-Effect of a spiral arm on accelerations of low-velocity masers in the NGC 4258 disk. (a) Initial conditions for an $N$-body simulation of a maser clump (triangle) orbiting a supermassive black hole of $3.8 \times 10^{7} M_{\odot}$ (Herrnstein et al. 2005) (star). The parameters of the trailing logarithmic spiral arm (gray), represented by 8000 particles, were arbitrarily chosen to be $r_{0}=1.5 \mathrm{mas}, b=0.42$, and $\phi_{0}=0^{\circ}$, where $r=r_{0} \exp \left[b\left(\phi-\phi_{0}\right)\right]$. The pattern speed of the spiral arm is half that of the Keplerian orbital velocity at any given radius. Note that the true breadth of the arm $\left(10^{-2} \mathrm{pc}\right)$ is exaggerated for clarity. (b) Snapshot at $t=100$ yr. $(c)$ Snapshot at $t=200 \mathrm{yr}$. (d) Maser component LOS acceleration as a function of disk impact parameter due to both the supermassive black hole and a spiral arm of varying masses. Arm mass is quoted as a percentage of the upper limit maser disk mass, $M_{\text {disk.upper }}=9 \times 10^{5} M_{\odot}$ (Herrnstein et al. 2005). Note the significant deviation of the acceleration profiles at $7 \%, 15 \%$, and $30 \%$ from that of the $0 \%$ (black hole only) sinusoid. The curve that best matches observations is that for an arm mass of $15 \%$. The gray hatched area indicates the observed projected extent in disk impact parameter of the low-velocity masers.

radial orbital velocity component is perpendicular to the LOS), the Doppler velocity is given by

$$
v_{\mathrm{los}}=\left(\frac{G M}{r}\right)^{1 / 2}[1+e \cos (\phi-\omega)]^{1 / 2} \cos \phi+v_{\mathrm{sys}},
$$

where $r$ is the angular radius in the disk and $M=M_{\mathrm{BH}} / D$. We can therefore compute the ratio of $v_{\text {red,los }} / v_{\text {blue,los }}$ for closed orbit $(e<1)$ values of $e$ and $\omega$ using $\phi=0^{\circ}$ for the redshifted highvelocity emission and $\phi=180^{\circ}$ for the blueshifted high-velocity emission. Note that this ratio is always unity for circular orbits $(e=0)$ and for orbits of any eccentricity viewed along the semimajor axes, i.e., for $\omega=90^{\circ}$ or $270^{\circ}$ in our definition. Using values of $v_{\text {sys }}=472 \pm 4 \mathrm{~km} \mathrm{~s}^{-1}$ (Cecil et al. 1992) and a disk dynamical center position of $x_{0}=-0.1 \pm 0.1$ mas estimated from jet continuum data (Herrnstein 1997) within the $1 \sigma$ uncertainties (noting that by allowing a $1 \sigma$ deviation in each quantity, we are actually using a combined uncertainty $>1 \sigma$ ), we computed the observed ratio of $v_{\text {red,los }} / v_{\text {blue,los }}$ from our VLBI data to lie between 0.98 and 1.03. This constrains maser orbits to have either a low eccentricity of $<0.05$ or to be more highly eccentric but viewed (nearly) along the semimajor axes of the orbits $\left(\omega=90^{\circ}\right.$ or $\left.270^{\circ}\right)$. The parameter space we eliminate using the PV diagram is marked by the white hatching of positive gradient in Figure 20.

To further constrain the eccentricity and periapsis angle, we can also use the low-velocity acceleration data as a function of Doppler velocity. Instantaneous acceleration is given by $\dot{v}_{\text {los }}=$ $-\left(G M / r^{2}\right) \sin \phi$ and $r=a_{\text {semi }}\left(1-e^{2}\right) /[1+e \cos (\phi-\omega)]$, so we can write

$$
\frac{d \dot{v}_{\mathrm{los}}}{d v_{\mathrm{los}}}=\left[\frac{G M}{\left(1-e^{2}\right)^{4} a_{\mathrm{semi}}^{3}}\right]^{1 / 2} \frac{\gamma^{2} \cos \phi-2 \gamma e \sin (\phi-\omega) \sin \gamma}{\sin \phi},
$$

where $\gamma=1+e \cos (\phi-\omega)$ and we assume that low-velocity masers occupy the same orbit, such that $a_{\text {semi }}$ is a constant implied by the restriction of low-velocity masers to a narrow portion of the PV diagram. Using the sign of the gradient, we can further exclude all periapsis angles of $90^{\circ} \leq \omega \leq 270^{\circ}$, marked by the white hatching of negative gradient in Figure 20. Since the $e-\omega$ 


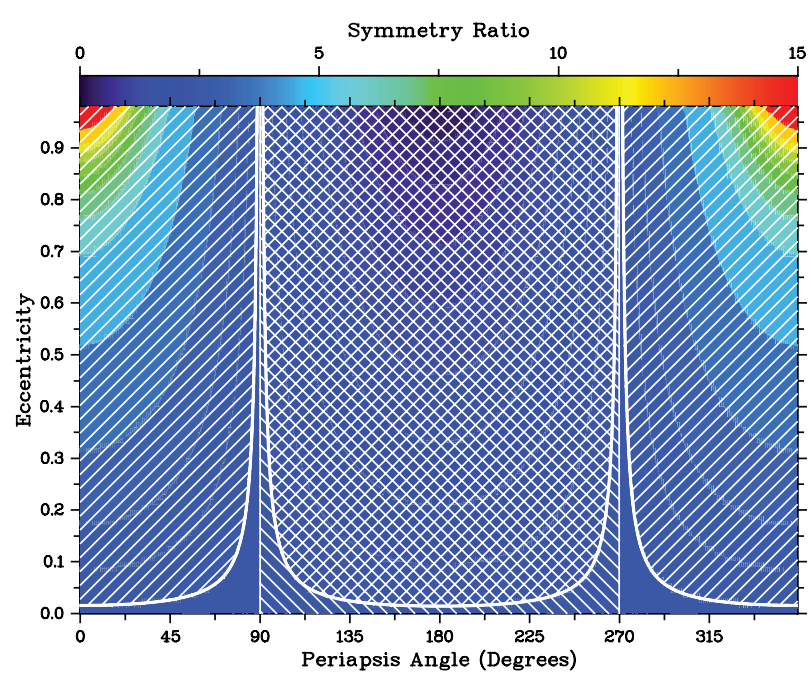

FIG. 20.-Contour plot of the "symmetry" ratio $\left(v_{\text {red,los }} / v_{\text {blue,los }}\right)$ of model PV diagrams for eccentricity $(e)$ as a function of periapsis angle $(\omega)$. The symmetry of the data eliminates values of $e$ and $\omega$ in the positive-gradient hatched regions of the plot. Using acceleration data for low-velocity emission, we further eliminate all of the negative-gradient hatched region of $90^{\circ} \leq \omega \leq 270^{\circ}$ for low-velocity maser components on the same orbit. Only completely unhatched regions of parameter space (neither hatched nor cross-hatched) are permitted by our data set.

parameter space remaining after these eliminations is very small (regions with no hatching at all in Fig. 20), it is unlikely that the maser orbits are highly eccentric and observed along a special viewpoint. However, this is a topic we revisit in the next paper of this series, in which we perform 3D accretion disk modeling of the masers including eccentric maser orbits.

\section{CONCLUSIONS}

In this paper we measured centripetal accelerations of maser spectral components for data spanning 1994 to 2004. We found that high-velocity emission accelerations lie in the range -0.7 to $+0.7 \mathrm{~km} \mathrm{~s}^{-1} \mathrm{yr}^{-1}$, indicating the emission originates within $13^{\circ}$ of the disk midline for material in Keplerian rotation (see Tables 2 and 3). The good agreement of maser projected vertical positions ( $y$-positions) of high-velocity emission derived from the acceleration data with those of VLBI images confirms that masers trace true gas dynamics of the disk. While the high-velocity accelerations do not support the MM98 model of trailing shocks associated with spiral arms in the disk, we find a spatial periodicity in high-velocity emission of wavelength 0.75 mas. This supports the model of Maoz (1995) of spiral structure due to density waves in the disk.

We measured accelerations of low-velocity emission in the range $7.7-8.9 \mathrm{~km} \mathrm{~s}^{-1} \mathrm{yr}^{-1}$, which is consistent with emission that originates from a concavity in the front side of the disk reported by Herrnstein et al. (2005). We confirm a systematic trend in accelerations of low-velocity emission as a function of component Doppler velocity, as found by Haschick et al. (1994) and Greenhill et al. (1995b). Preliminary investigations into the origin of the trend suggest that eccentricity in maser orbits is unlikely to be the cause. The trend may be caused either by the effect of a stationary or slowly moving spiral arm, or by a disk feature that causes a stationary pattern of radius versus azimuth angle.

We are grateful to Maryam Modjaz and Paul Kondratko for providing their GBT data. We thank Alar Toomre for helpful discussions.

Aarseth, S. J. 1985, in IAU Symp. 113, Dynamics of Star Clusters, ed. J. Goodman \& P. Hut (Dordrecht: Reidel), 251

Argon, A. L., Greenhill, L. J., Reid, M. J., Moran, J. M., \& Humphreys, E. M. L. 2007, ApJ, 659, 1040 (Paper I)

Benedict, G. F., et al. 2002, AJ, 124, 1695

Bogdanović, T., Eracleous, M., Mahadevan, S., Sigurdsson, S., \& Laguna, P. 2004, ApJ, 610, 707

Bragg, A. E., Greenhill, L. J., Moran, J. M., \& Henkel, C. 2000, ApJ, 535, 73

Caputo, F., Marconi, M., \& Musella, I. 2002, ApJ, 566, 833

Cecil, G., Wilson, A. S., \& Tully, R. B. 1992, ApJ, 390, 365

Claussen, M. J., Heiligman, G. M., \& Lo, K. Y. 1984, Nature, 310, 298

Cooke, B., \& Elitzur, M. 1985, ApJ, 295, 175

Freedman, W. L., et al. 2001, ApJ, 553, 47

Genzel, R., et al. 2003, ApJ, 594, 812

Greenhill, L. J., Henkel, C., Becker, R., Wilson, T. L., \& Wouterloot, J. G. A. 1995a, A\&A, 304, 21

Greenhill, L. J., Jiang, D. R., Moran, J. M., Reid, M. J., Lo, K. Y., \& Claussen, M. J. 1995b, ApJ, 440, 619

Haschick, A. D., \& Baan, W. A. 1990, ApJ, 355, L23

Haschick, A. D., Baan, W. A., \& Peng, E. W. 1994, ApJ, 437, L35

Herrnstein, J. R. 1997, Ph.D. thesis, Harvard Univ.

Herrnstein, J. R., Greenhill, L. J., \& Moran, J. M. 1996, ApJ, 468, L17

Herrnstein, J. R., Moran, J. M., Greenhill, L. J., \& Trotter, A. S. 2005, ApJ, 629,719

Herrnstein, J. R., et al. 1999, Nature, 400, 539

Hu, W. 2005, in ASP Conf. Ser. 339, Observing Dark Energy, ed. S. C. Wolff \& T. R. Lauer (San Francisco: ASP), 215

Jensen, J. B., Tonry, J. L., Barris, B. J., Thompson, R. I., Liu, M. C., Rieke, M. J., Ajhar, E. A., \& Blakeslee, J. P. 2003, ApJ, 583, 712

Macri, L. M., Stanek, K. Z., Bersier, D., Greenhill, L. J., \& Reid, M. J. 2006, ApJ, 652, 1133

Maoz, E. 1995, ApJ, 455, L131

Maoz, E., \& McKee, C. F. 1998, ApJ, 494, 218

Miyoshi, M., Moran, J., Herrnstein, J., Greenhill, L., Nakai, N., Diamond, P., \& Inoue, M. 1995, Nature, 373, 127

Modjaz, M., Moran, J. M., Kondratko, P. T., \& Greenhill, L. J. 2005, ApJ, 626, 104

Moran, J., Greenhill, L., Herrnstein, J., Diamond, P., Miyoshi, M., Nakai, N., \& Inque, M. 1995, Proc. Natl. Acad. Sci., 92, 11427

Moran, J. M., Greenhill, L. J., \& Herrnstein, J. R. 1999, J. Astrophys. Astron., 20,165

Nakai, N., Inoue, M., Miyazawa, K., Miyoshi, M., \& Hall, P. 1995, PASJ, 47, 771

Nakai, N., Inoue, M., \& Miyoshi, M. 1993, Nature, 361, 45

Newman, J. A., Ferrarese, L., Stetson, P. B., Maoz, E., Zepf, S. E., Davis, M., Freedman, W. L., \& Madore, B. F. 2001, ApJ, 553, 562

Sandage, A., Tammann, G. A., Saha, A., Reindl, B., Macchetto, F. D., \& Panagia, N. 2006, ApJ, 653, 843

Spergel, D. N., et al. 2007, ApJS, 170, 377

Statler, T. S. 2001, AJ, 122, 2257

Toomre, A. 1964, ApJ, 139, 1217

. 1981, in Structure and Evolution of Normal Galaxies, ed. S. M. Fall \& D. Lynden-Bell (Cambridge: Cambridge Univ. Press), 111

Udalski, A., Wyrzykowski, L., Pietrzynski, G., Szewczyk, O., Szymanski, M., Kubiak, M., Soszynski, I., \& Zebrun, K. 2001, Acta Astron., 51, 221

Watson, W. D., \& Wallin, B. K. 1994, ApJ, 432, L35

Yamauchi, A., Sato, N., Hirota, A., \& Nakai, N. 2005, PASJ, 57, 861 